DOI: $10.3901 / J M E .2021 .21 .248$

\title{
智能刀具研究综述
}

\author{
刘 强 $1,2,3$ 张海军 ${ }^{1,2}$ 刘献礼 ${ }^{1,2}$ 高大湧 ${ }^{1,2}$ 张明鉴 ${ }^{1,2}$
}

(1. 哈尔滨理工大学先进制造智能化技术教育部重点实验室 哈尔滨 150080 ;

2. 哈尔滨理工大学高效切削及刀具国家地方联合工程实验室 哈尔滨 150080 ;

3. 哈尔滨理工大学电气工程博士后流动站 哈尔滨 150080)

\begin{abstract}
摘要: 智能刀具根据加工中具体用途的不同, 可实现对切削状态在线监测、数据处理、切削过程优化控制等功能, 通过智能 刀具的使用可改善加工过程, 提高加工质量与效率, 到目前为止学者们对于智能刀具的研究已取得大量研究成果。对智能刀 具切削状态监测和切削过程控制两个方面的研究进展进行论述, 梳理了学者们应用智能刀具对切削力、切削温度、刀具振动 进行监测与控制的研究成果, 对刀具结构、监测方式、控制原理、缺点不足、发展方向进行了总结与讨论。对智能刀具涉及 的关键技术进行探讨, 由于智能刀具涉及多学科交叉, 实现的功能及采用的原理各不相同, 关键技术多样, 需进行多学科交 叉融合，并通过产学研协同合作，推进智能刀具关键技术的深入研究及实际应用。
\end{abstract}

关键词: 智能刀具; 切削状态监测; 切削过程控制; 切削力; 切削温度; 刀具振动

中图分类号: TG71

\section{A Review of Research on Intelligent Cutting Tools}

\author{
LIU Qiang ${ }^{1,2,3}$ ZHANG Haijun ${ }^{1,2}$ LIU Xianli1 ${ }^{1,2}$ GAO Dayong ${ }^{1,2}$ ZHANG Mingjian ${ }^{1,2}$
}

(1. Key Laboratory of Advanced Manufacturing and Intelligent Technology, Ministry of Education, Harbin University of Science and Technology, Harbin 150080;

2. The Lab of National and Local United Engineering for High-Efficiency Cutting \& Tools, Harbin University of Science and Technology, Harbin 150080;

3. Postdoctoral Research Station of Electrical Engineering, Harbin University of Science and Technology, Harbin 150080)

\begin{abstract}
According to the different specific uses in the processing, the intelligent cutting tools can realize on-line monitoring, data processing, optimization control of cutting process and other functions. The use of intelligent cutting tools can improve the machining process, and improve the processing quality and efficiency. Up to now, a lot of research achievements have been made. The research progress of intelligent cutting tools in cutting state monitoring and cutting process control is discussed. The research results of monitoring and controlling cutting force, cutting temperature and tool vibration by using intelligent cutting tools are summarized. The structures, monitoring methods, control principles, shortcomings and development direction of the intelligent cutting tools are summarized and discussed. The key technologies involved in intelligent cutting tools are discussed. As intelligent cutting tools involve multidisciplinary crossing, their functions and principles are different, and their key technologies are diverse, it is necessary to carry out multidisciplinary cross fusion, and promote the in-depth research and practical application of key technologies of intelligent cutting tools through industry-university-research cooperation.
\end{abstract}

Key words: intelligent cutting tools; cutting condition monitoring; cutting process control; cutting force; cutting temperature; tool vibration

\footnotetext{
* 国家自然科学基金(51805122)、黑龙江省博士后(LBH-Z18100)、国家自然 科学基金国际(地区)合作与交流(51720105009)资助项目。20201109 收到 初稿, 20210911 收到修改稿
} 


\section{0 前言}

随着新一轮产业革命的兴起, 加速了工业智能 化和绿色化的转型, 促进不同产业相互融合, 智能 制造作为信息化和工业化高度融合的新兴领域, 得 到各国的普遍重视, 如德国 “工业 4.0”、“中国制造 2025 ” 等规划的出台都推动了智能制造的发展 ${ }^{[1]}$ 。 切削加工在机械制造中占据重要地位, 占机械加工 的 $90 \%$ 以上 ${ }^{[2]}$, 它是促进航空航天、汽车工业、新 能源、电子及模具等行业制造技术发展的重要因素。

刀具作为切削加工中的工具终端, 是机床的“牙 齿”, 常规刀具的功能集中在 “切削” 范畴, 通过使 用刀具对金属等材料进行去除加工, 围绕刀具的研 究包括刀具的设计及制造, 涉及的具体研究内容主 要包括: 刀具材料 ${ }^{[3]}$ 、涂层 ${ }^{[4]}$ 、刀具角度 ${ }^{[5]}$ 、刃磨 ${ }^{[6]}$ 、 刀具磨破损 ${ }^{[7]}$ 等。但随着智能刀具的提出及发展, 使对刀具的研究不再仅仅局限于 “切削” 功能, 而 是提出了可对切削状态进行监测的 “感知型” 刀具, 对切削过程进行优化控制的 “受控型” 刀具, 对切 削过程数据进行存储、分析、挖掘、学习的 “学习 型”刀具等。

近年来, 国内外学者对智能刀具开展了大量的 研究工作, 主要针对的智能刀具为 “感知型” 刀具 和 “受控型” 刀具。切削加工中, 切削力、切削温 度以及刀具振动对切削过程及最终加工质量影响显 著, “感知型” 刀具通过对以上物理量的状态变化进 行监测及分析, 可对切削过程是否顺利进行有效的 判断, 从而对实际生产加工进行指导, 所获得的监 测信号为进一步实现刀具控制提供了反馈信息。 “受控型” 刀具通过传感器获得切削状态信息, 控制 系统根据所获得的状态信息及控制算法对 “受控型” 刀具的相关参数进行调节, 从而实现对切削过程的 调控, 优化加工过程。

现有智能刀具的自动化程度已经比较完备, 具 有感知功能、自调控功能, 是 “弱智能” 刀具。对 于 “学习型” 刀具的研究相对较少, 需要结合工业 互联网、大数据、云计算、人工智能等多个领域的 研究成果, 对 “学习型” 刀具开展深入研究, 最终 提出具有 “强智能” 的智能化刀具。

\section{1 智能刀具介绍}

智能刀具是相对于传统刀具而言的概念, 传统 刀具只能按照机床预先设定的切削参数和切削路线
进行走刀, 在加工过程中无法实现对切削状态的 感知及自身切削性能的调控, 仅具有 “切削” 功 能。智能刀具则根据研究侧重点的不同, 可单独 或同时具有对切削状态感知, 自身切削性能调 控, 加工数据学习的功能, 作者对智能刀具的分 类如图 1 所示。

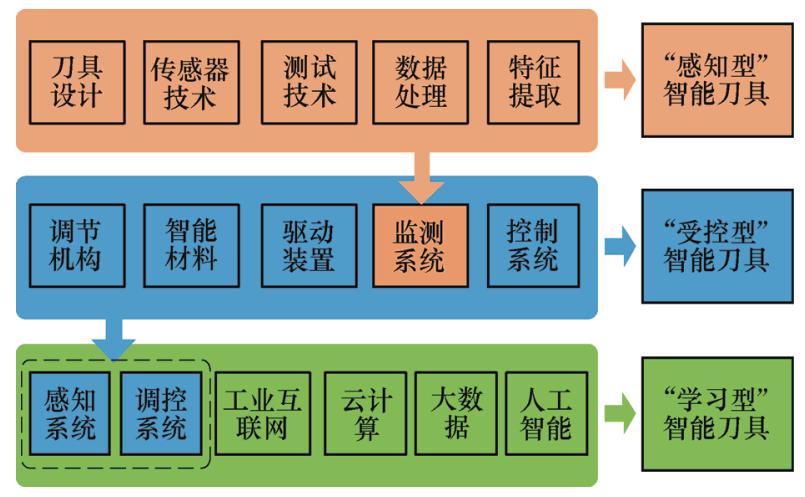

图 1 智能刀具分类

“感知型” 智能刀具通过对刀具结构进行改进设 计, 使刀具适合不同传感器的安装, 从而实现对切 削加工中切削力、切削热、振动等的测量, 通过数 据处理及特征提取对切削状态进行表征, 实现对切 削过程的状态监测。

“受控型”智能刀具通过在刀具外部布置监测系 统或通过刀具自身的监测系统实现对加工状态的监 测, 其控制系统通过对监测数据的分析, 结合控制 算法及驱动装置对刀具的切削性能进行在机调控, 优化加工过程及加工质量和效率。

“学习型” 智能刀具是伴随工业互联网、大数据、 云计算、人工智能技术的发展而产生的, 在刀具具 备感知系统与调控系统的基础上, 利用工业互联网、 大数据、云计算、人工智能等技术, 完成智能刀具 对数据储存、分析、挖掘、学习等功能, 真正使智 能刀具变的 “聪明”。

\section{2 智能刀具切削状态监测技术}

切削状态监测是实现智能加工的必备功能, 切 削状态实时监测对于控制切削过程、调整切削参数、 监测刀具磨破损、保证加工质量等具有重要作用, 能够有效提高加工精度和保障设备安全 ${ }^{[8]}$ 。通过在 智能刀具上集成测量系统, 可较方便地对切削过程 进行实时监测, 实现刀具自身对切削状态的 “自感 知”, 同时避免了在机床上加装复杂的监测系统, 降 低监测成本, 在实际生产中易于实现, 目前已经取 得较多的研究成果 ${ }^{[9]}$ 。 


\section{1 切削力自感知刀具}

通过切削力对切削状态进行监测是最直接、最 有效的方式之一, 切削力对切削状态变化的响应速 度快, 并且切削力的大小直接对切削温度、加工振 动、刀具磨损、机床功率等产生影响。因此, 对切 削力的测量十分重要, 目前主要的切削力测量刀具 包括: 应变式切削力测量刀具 ${ }^{[10-12]}$ 、压电式切削力 测量刀具 ${ }^{[13-14]}$ 、电容式切削力测量刀具 ${ }^{[15]}$ 和基于声 表面波原理的切削力测量刀具 ${ }^{[16]}$ 。

(1) 应变式切削力测量刀具。

应变式切削力测量刀具主要由弹性元件、电阻 应变片及相应的测量转换电路组成, 弹性元件受力 会引起电阻应变片电阻值的变化, 进而影响输出电 压变化, 通过输出电压与力之间的关系可求得切削 力的大小。

麻省理工学院于 20 世纪 50 年代率先将电阻式 应变片应用于力的测量; 哈尔滨工业大学袁哲俊教 授所带领的团队于 60 年代进行了系列化应变式切 削力测量刀具的研究。由于应变式切削力测量刀具 具有较好的研究基础, 且结构灵活多变, 依然是切 削力测量刀具的重要研究方向。

SÜLEYMAN 等 ${ }^{[17]}$ 研制了一种用于车削的集成 化切削力测量刀具, 如图 2 所示。通过 4 个八角应 变环组合结构实现对三向切削力的测量, 其测量极 限为 $3500 \mathrm{~N}$, 系统灵敏度为 $\pm 5 \mathrm{~N}$, 对该测力仪进行 了静态和动态标定, 整个装置体积相对较大, 但充 分体现了测力装置与刀具集成的思想。通过实验数 据分析，表明该测力仪能够可靠地应用于车削切削 力的测量。

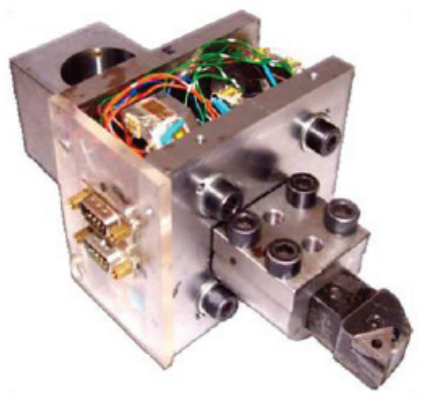

图 2 集成化切削力测量刀具 ${ }^{[17]}$

PANZERA 等 ${ }^{[18]}$ 设计了一种通过感知弹性元件 扭转和弯曲进而获得三向切削力的集成化刀具系 统。将应变片粘贴于弹性元件上, 弹性元件用于固 定刀具, 最终实现三向切削力的解耦。所能感知的 最小切削力为 $5 \mathrm{~N}$, 整个系统结构较为简单, 但体 积依然较大, 刀具具体结构如图 3 所示。

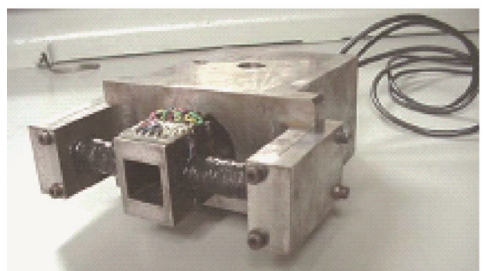

图 3 三向切削力测量刀具系统 ${ }^{[18]}$

赵友等 ${ }^{[19]}$ 同样提出了集成化的切削力测量刀 具, 将车刀安装在相互垂直的八角环弹性套筒中, 在八角环弹性套筒的表面粘贴电阻应变片, 实现对 三向切削力的测量, 如图 4 所示。该传感器测量静 态外力的误差在 $0.38 \% \sim 0.83 \%$ 范围内, 三个方向的 测量输出特性分别为: 主切削力方向为 $1.06 \times 10^{-2} \mathrm{mV} / \mathrm{N}$, 进给力方向为 $1.14 \times 10^{-2} \mathrm{mV} / \mathrm{N}$, 而轴向力方向为 $0.18 \times 10^{-2} \mathrm{mV} / \mathrm{N}$, 此种结构进一步 减小了集成化刀具的体积。

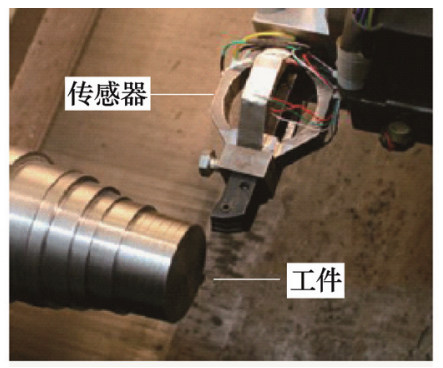

图 4 双八角环切削力测量刀具 ${ }^{[19]}$

在此基础上，为进一步缩小集成化刀具体积， 赵友等 ${ }^{[20]}$ 提出以刀杆本体作为弹性敏感元件、以半 导体应变计作为信号转换元件的切削力自感知智能 刀具, 如图 5 所示。在刀具前端开设凹槽, 将半导 体应变计嵌入凹槽中, 从而使整个刀具结构极为紧 凑, 试验结果显示主切削力 $F_{\mathrm{c}}$ 方向的静态精度为 $1.799 \%$ 、进给力 $F_{\mathrm{f}}$ 方向的静态精度为 $1.628 \%$, 刀 具的固有频率为 $1778.98 \mathrm{~Hz}$, 可以满足在机床主轴 转速不超过 $26685 \mathrm{r} / \mathrm{min}$ 的切削力测量需求。

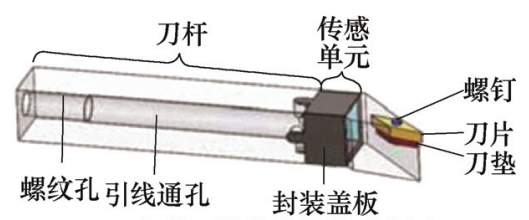

（a）智能刀具整体结构示意图

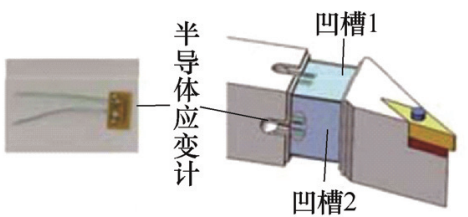

(b) 智能刀具整体结构示意图

图 5 应变式切削力自感知智能刀具 ${ }^{[20]}$ 
HARMON 等 ${ }^{[21]}$ 研制一种基于蓝牙传输的无线 智能刀柄, 结构如图 6 所示。应变片直接粘贴于刀 柄的内部, 数据传输采用蓝牙无线传输方式, 将信 号传输和信号放大电路集成在电路板上, 并封装在 刀柄内部。通过对刀柄弯曲应变的测量求解出刀具 的径向切削力与切向切削力的合力, 与商用测力仪 所获得的数据进行比较, 数据差异不大, 说明所提 出的智能刀柄具有较好的测量精度。

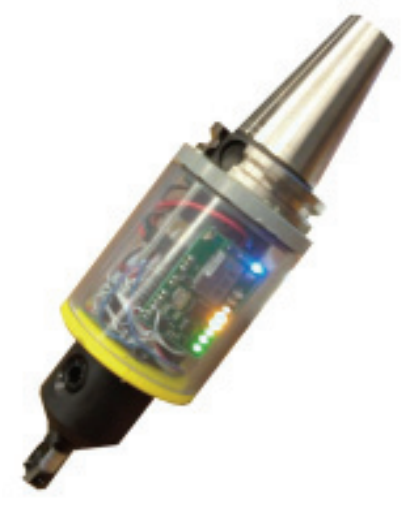

图 6 无线传输智能刀柄 ${ }^{[21]}$

RIZAL 等 ${ }^{[22]}$ 研制一种基于应变片的旋转测力 刀柄, 如图 7 所示。在刀柄与刀具的连接处设计了 力传感器, 力传感器由四个均布的 L 形梁式结构构 成, 每根梁上布置 6 个应变片, 通过程序解算, 该 装置能够测量主切削力、进给力以及垂直方向的切 削力, 测量极限为 $3000 \mathrm{~N} 、 5000 \mathrm{r} / \mathrm{min}$, 可完成铣 削、钻削加工中切削力的测量。

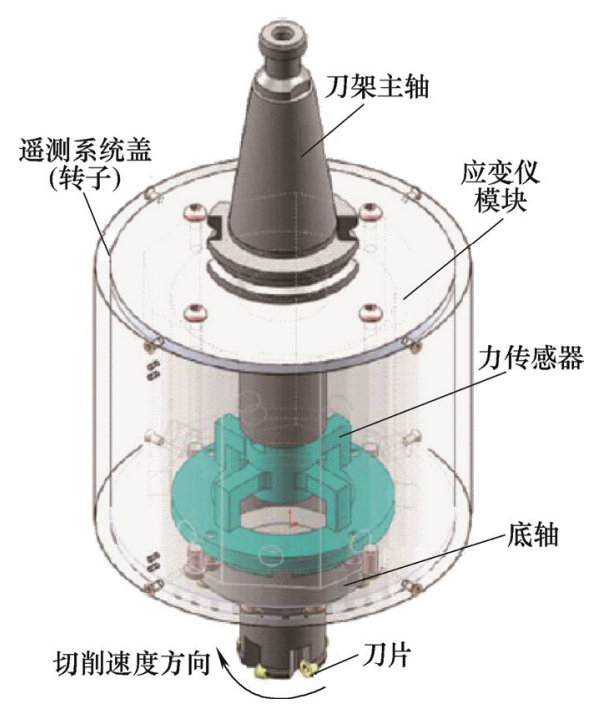

图 7 旋转测力刀柄 ${ }^{[22]}$

SUPROCK 等 ${ }^{[23]}$ 则是直接将应变片贴于刀体 上, 刀体与刀柄、信号采集模块、无线传输模块系 统集成在一起。应变片数据线通过刀体的冷却孔连 接至刀体后端, 通过 DIN 航空插头与刀柄系统的信
号采集和无线传输模块相连接, 可实现对切削力、 扭矩、温度的采集, 该系统采用蓝牙传输方式, 信号传输较为方便, 集成式切削力测量刀具系统如 图 8 所示。

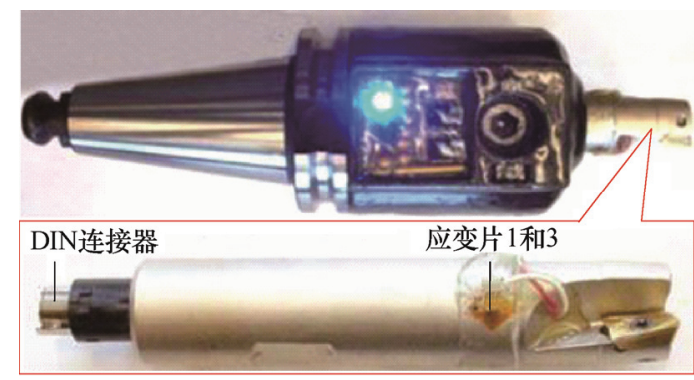

图 8 集成式切削力测量刀具系统 ${ }^{[23]}$

德国 Pro-micron 公司基于应变式传感器开发了 商用化的 Spike 无线动态切削力测量系统, 不同于 以往的三向切削力测量, Spike 无线动态切削力测量 系统可同时在线监测刀具轴向力、扭矩、弯矩、温 度多种信号, 系统如图 9 所示。将其配合机床使用 能够实现加工状态实时监测, 评估刀具磨损、破损 状态。

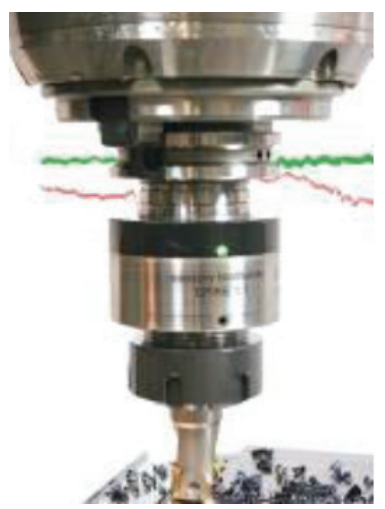

图 9 Spike 无线动态切削力测量系统

由以上可知, 应变式切削力测量刀具具有结构 灵活、适应性广、技术积累深厚等优点, 且随着应 变式传感器配套仪器设备标准化程度不断完善, 使 得应变式切削力测量刀具将会得到广泛应用。考虑 到其测量精度和动态特性由弹性元件结构所决定, 如何解决灵敏度和刚度之间的矛盾, 是提高应变式 切削力测量刀具测量精度的关键。

(2) 压电式切削力测量刀具。

1963 年德国阿亨工业大学首先研制成功了采 用压电晶体作为传感器的三向车削力测量装置 ${ }^{[24]}$ 。 我国大连理工大学孙宝元教授所带领的团队对压电 式切削力测量装置开展了大量研究工作, 并成功研 制了多种可靠实用的压电式切削力测量装置 ${ }^{[25-27]}$, 取得了良好工程应用效果。随着国内外学者研究的 
不断深入, 压电式切削力测量刀具取得了丰富的研 究成果。

孙宝元教授课题组研制了系列化的切削力测量 刀具, 图 10 为其团队所研制的 YDC-III89 型刀杆式 压电动态切削力测量刀具 ${ }^{[28]}$, 在半环式弹性体内设 置三向压电晶体来感知三向切削力, 为使刀具在 $X$ 、 $Y 、 Z$ 三个方向刚度近似相等且保证各向具有较大的 固有频率, 对半环式弹性体进行了结构优化。该方 案结构较为紧凑、简单, 使用方便, 实用性较强。

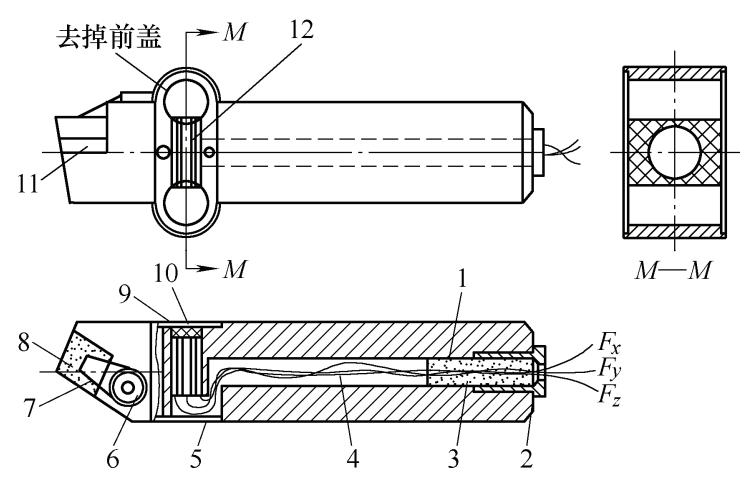

图 10 刀杆式压电动态切削力测量刀具 ${ }^{[28]}$

肖才伟 ${ }^{[29]}$ 提出一种三向切削力感知式智能刀 具, 结构如图 11 所示。将四片压电陶瓷传感器集成 到具有凹腔感知区的刀杆前端, 通过分析压电陶瓷 的电荷分布、大小及其与作用力的映射关系, 建立 了压电陶瓷输出电荷与三向切削力的耦合关系, 实 现三向切削力求解, 最终通过切削实验, 验证了切 削力解耦的正确性, 实现了三向切削力在线测量。 所提出的智能切削力测量刀具整体结构紧凑。

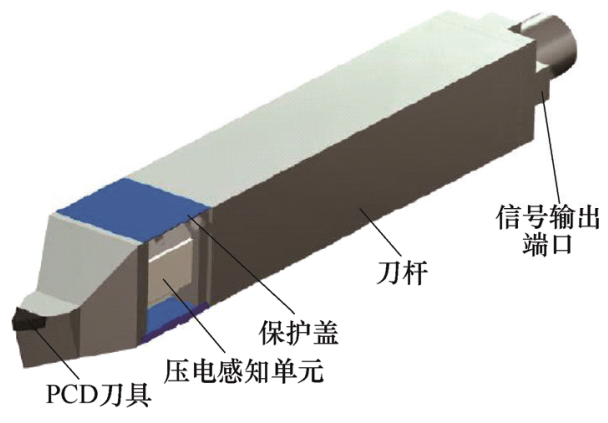

图 11 三向切削力感知式智能刀具 ${ }^{[29]}$

TOTIS 等 ${ }^{[30]}$ 提出了在刀柄前端加装 Kistler9251A 型三向力传感器的模块化切削力测量 刀具, 如图 12 所示。通过所提出的刀柄结构, 可在 不拆卸力传感器的情况下轻松更换切削刀片。此种 方式将商用化传感器与刀具进行结构集成, 通过简 单的结构改造实现对切削力的测量。但此种方式改 变了机床的原有部件, 配套测量系统价格昂贵, 在
实际大生产应用中推广使用存在一定困难。

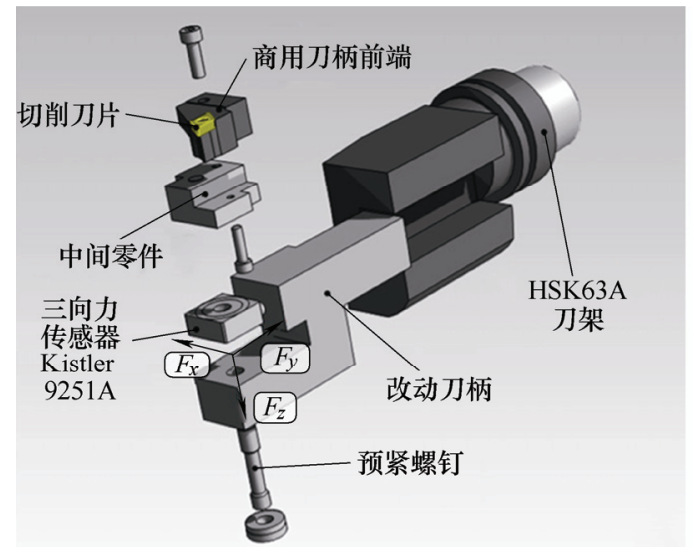

图 12 模块化切削力测量刀具 ${ }^{[30]}$

同时, TOTIS 等 ${ }^{[31]}$ 研制了一种用于测量面铣刀 单个铣刀片切削力的智能刀具系统, 如图 13 所示。 每个铣削刀片的固定端都安装商用的 Kistler9251A 型三向力传感器, 可从每个切削刀片上测量出独立 的三向切削力。由于是旋转刀具, 在刀柄上配备了 高性能的数据传输遥测系统, 所获得的信号通过放 大、低通滤波处理, 最终实现对铣削方式切削力的 测量。

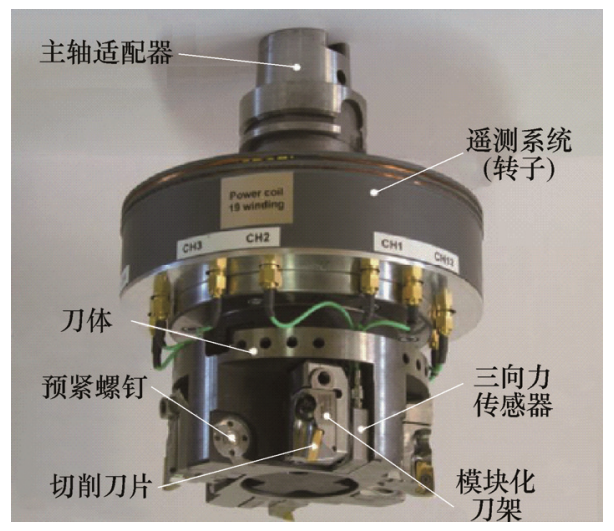

图 13 面铣刀切削力测量刀具系统 ${ }^{[31]}$

任宗金等 ${ }^{[32-33]}$ 研制一种可安装在机床主轴上的 刀柄式压电切削测力仪, 如图 14 所示。在刀柄传感 器内部安装了一组 $X$ 切型和一组 $Y$ 切型石英晶片及 轻型深沟球轴承, 分析了晶片在三向切削力下电荷 分布情况, 采用三个轻型深沟球轴承对轴向力, 径 向力和扭矩电荷信号进行输出, 实现了切削力和扭 矩的测量。

利用压电薄膜的压电效应, 同样可以实现对切 削力的测量, 并且压电薄膜具有体积小, 灵敏度高, 利于刀具系统集成化等特点, 在切削力的测量上已 多有应用。

ZHENG 等 ${ }^{[34]}$ 将 AlN 压电薄膜涂覆在切削刀片 
底部形成感知单元, 为了防止发生短路, 在压电薄 膜外包裹了一层绝缘层。试验结果表明所构建的切 削力感知系统具有良好的灵敏度、线性信号响应能 力和较大的带宽, 同时, 发现压电薄膜对切削振动 信号有很好的感知能力。

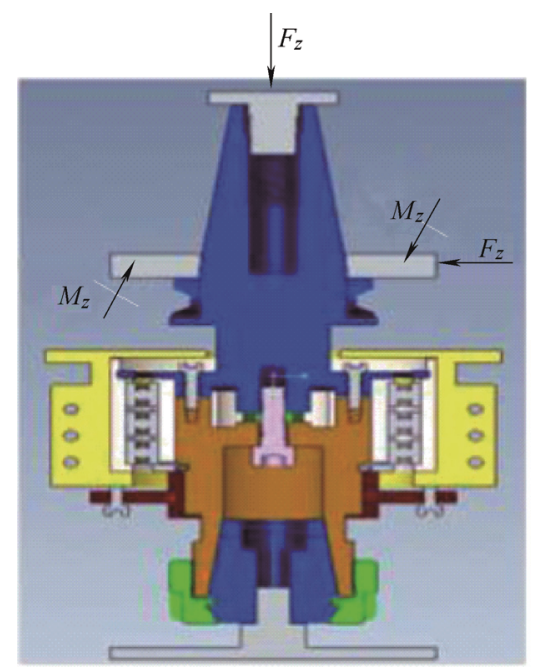

图 14 刀柄式压电切削测力仪 ${ }^{[32]}$

程凯教授团队 ${ }^{[35-36]}$ 设计了一种集成压电薄膜传 感器的测力刀具, 如图 15 所示。将压电薄膜传感器 设置在硬质合金刀片与刀垫之间, 并对压电薄膜施 加预载荷以保证测量的灵敏度, 最终实现对主切削 力的感知, 整个刀具系统结构紧凑, 集成化高。

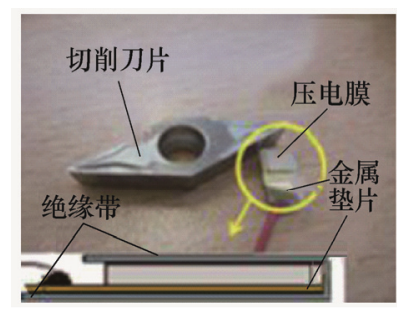

(a) 薄膜式测力刀片组成

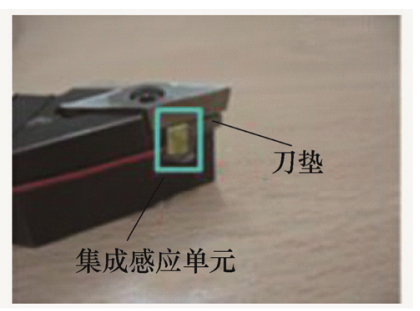

(b) 薄膜式测力刀片安装
图 15 薄膜式测力刀具 ${ }^{[35]}$

罗明等 ${ }^{[37]}$ 将 PVDF 压电薄膜嵌入在铣刀齿背后 三个接触面上, 实现铣削中每齿切削力的测量, 如 图 16 所示。在切削力作用下 PVDF 压电薄膜产生电 荷, 通过电荷放大器转换为电压信号, 进行 $\mathrm{AD}$ 转 换后采用无线传输方式至上位机进行分析处理。同 时, 所提出的内嵌薄膜传感器的仪器化铣刀系统也 可以识别刀具的磨损和成屑情况。

$\mathrm{MA}$ 等 ${ }^{[38-39]}$ 提出了基于 PVDF 压电薄膜的集成 化刀具系统, 用于监测端铣加工过程中的动态切削 力和扭矩。如图 17 所示, 通过在刀杆圆周布置 PVDF 压电薄膜拾取刀杆的动态剪切应变, 最终实现对 $X$ 、 $Y$ 向切削力和扭矩的测量。其中, 文献[39]所采用的
PVDF 传感器粘贴形式可较好地降低 PVDF 传感 器的热释电效应, 减小弯曲应变、热应变对测量 的影响。

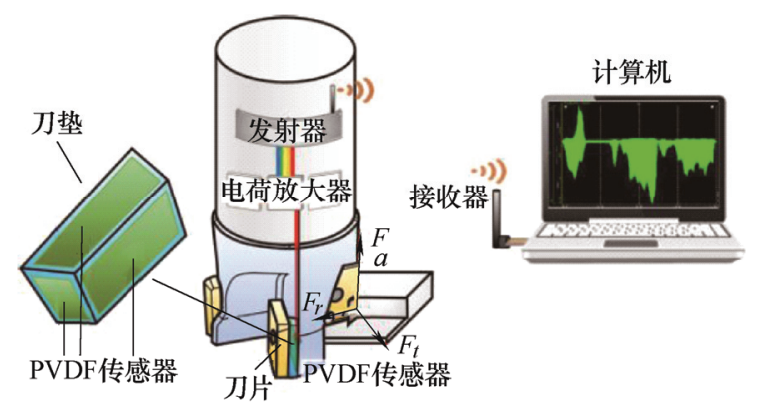

图 16 薄膜式铣削力测量系统 ${ }^{[37]}$

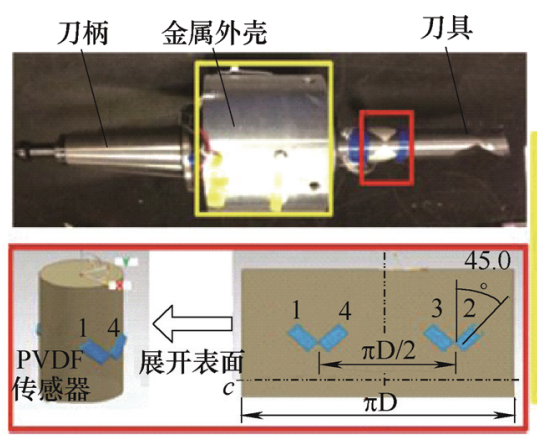

微控制器 微型SD卡

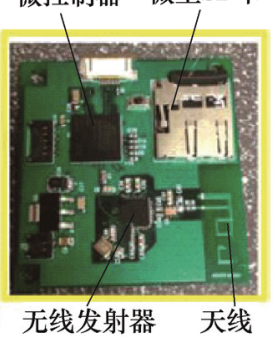

图 17 刀柄测力仪结构图 ${ }^{[38]}$

从动态切削力测量的角度考虑, 压电传感器是 较为理想的力感知元件, 但同时压电传感器也存在 如电荷泄露、不能测试静态力、维护不方便、价格 昂贵等缺点。尤其是薄膜压电传感器, 面临着切削 过程中切削热的严重影响, 导致信息失真和失效的 情况。但考虑到压电传感器具有高灵敏度, 受力变 形小等优点, 其依然是切削力测量的理想感知元件, 随着研究的持续深入及技术不断发展, 压电式切削 力测量刀具必定会得到广泛应用。

(3) 电容式切削力测量刀具。

电容式传感器采用非接触的方式对刀具的微变 形量进行测量, 将感受到的外界力的变化转换为电 容的变化, 刀具本身作为电容极板, 相对于其他智 能刀具, 其传感器的布置方式较为方便。

路勇教授团队 ${ }^{[40]}$ 提出了刀柄内部安装电容片 的四维测力刀柄, 对刀柄进行切槽形成弹性体, 在 刀柄上方宽槽的轴向、径向敏感位置粘贴 4 个电容 片, 在扭转敏感位置粘贴 2 个电容片, 依据形变位 置关系实现切削力的测量, 与商用测力仪 Kistler 9257B 所测量的切削力数值对比得到轴向力误差在 $13 \%$ 以内, 径向力误差在 $6.5 \%$ 以内。刀具整体结构 如图 18 所示。 


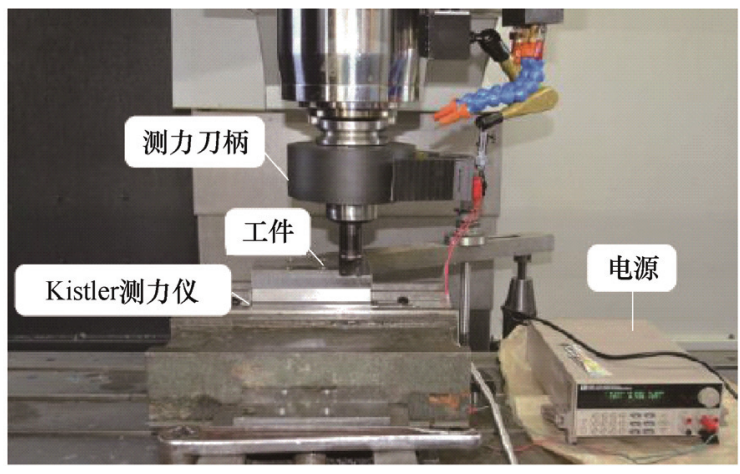

图 18 电容式测力刀柄 ${ }^{[40]}$

ALBRECHT 等 ${ }^{[41}$ 提出一种基于电容位移传感 器的铣削力测量系统, 将电容位移传感器安装于主 轴外端并进行集成, 具体结构如图 19 所示。通过测 量传感器测头与主轴之间间隙的变化间接测量切削 力, 分析了传感器的布置方式对测量的影响, 最终 完成了对径向切削力的测量。

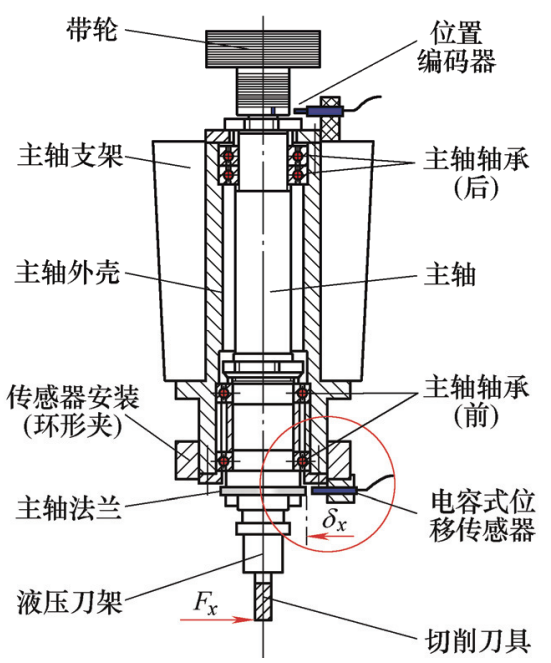

图 19 电容式铣削力测量系统 ${ }^{[1]}$

$\mathrm{KIM}$ 等 ${ }^{[42]}$ 提出了基于圆柱结构电容传感器的 测力刀具, 如图 20 所示。在主轴四周均布 4 个结构 为 $1 / 4$ 圆柱形电容传感器, 通过测量主轴在径向方 向的偏移实现 $X 、 Y$ 向切削力的测量, 实验结果表 明所提出的测力刀具可实现高转速下切削力的 测量。

电容式切削力测量刀具传感器的布置操作相对 简单, 可实现不降低刚度的情况下对切削力进行测 量, 多数是对径向力进行测量, 而对于轴向力的测 量难度较大, 存在一定局限性, 但电容式切削力测 量刀具依然是切削力测量刀具研究的重要方向 之一。

(4) 声表面波式切削力测量刀具。

声表面波是沿材料表面传播的一种弹性波，利 用声表面波测力的原理为当声表面波谐振器内部石
英基片受力后会产生应变，从而导致自身谐振频率 发生变化，通过对谐振频率偏移量的测量进而得到 作用力的大小。

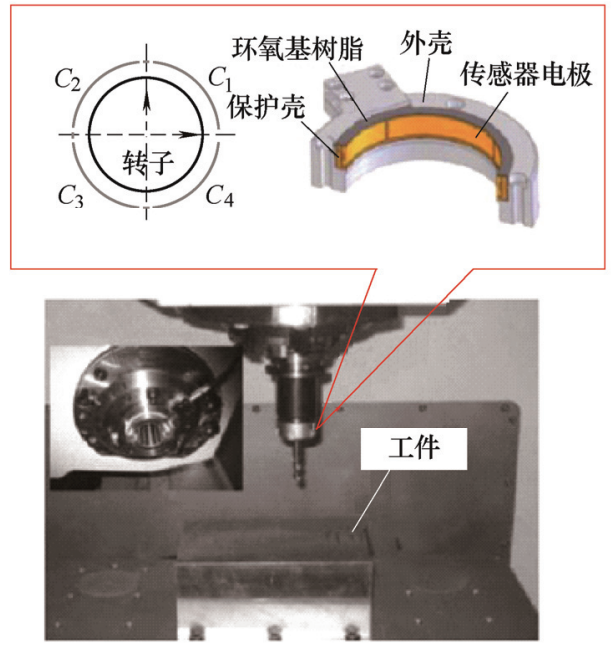

图 20 圆柱式电容测力刀具 ${ }^{[42]}$

STONEY 等 ${ }^{[43-44]}$ 提出了一种基于声表面波原理 的切削力测量刀具, 如图 21 所示, 将全封装的单端 口声表面波谐振应变传感器安装于刀具上表面, 利 用传感器的谐振频率与施加的应变成线性关系, 可 对切削过程的动态切削力进行测量。试验结果表明 该传感器对外加载荷具有高灵敏度和线性响应, 实 现了对主切削力的测量。随后 STONEY 等又提出了 安装 2 个声表面波谐振应变传感器的测力刀具, 实 现了 2 个方向上切削力的测量。

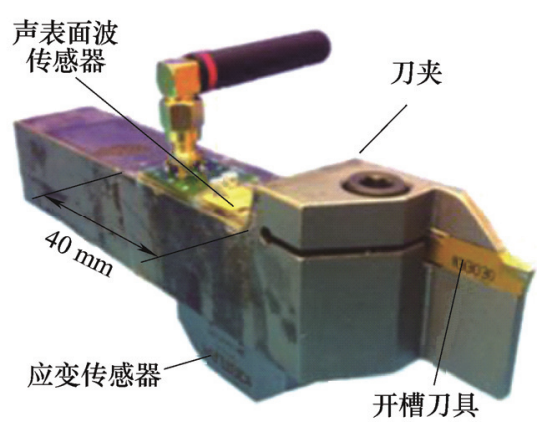

图 21 声表面波式切削力测量刀具 ${ }^{[43]}$

程凯团队 ${ }^{[45]}$ 同样提出了一种基于声表面波 原理的切削力测量刀具, 具体结构如图 22 所示。 将单个声表面波谐振器通过环氧树脂胶粘在车 刀刀杆上表面, 建立了切削力与声表面波谐振器 石英基片应变的关系模型, 得到了切削力与声表 面波谐振频率偏移量的关系, 实现了对车削加工 主切削力 $20 \sim 200 \mathrm{~N}$ 的实时测量。试验结果表明 作者所提出的切削力测量刀具具有较好的测量 准确性与动态性能。 


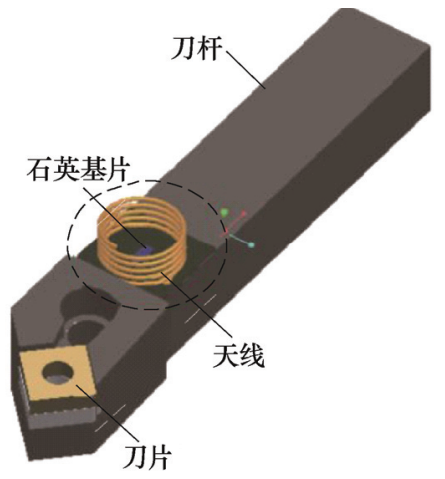

图 22 声表面波车削测力刀具 ${ }^{[45]}$

WANG 等 ${ }^{[46]}$ 将两个声表面波应变传感器分别 安装在刀柄前端的上表面和侧面, 实现了对主切削 力和进给力的测量。其中主切削力和进给力测量迟 滞分别为 $7.3 \%$ 和 $4.7 \%$, 向间干扰分别为 $20.3 \%$ 和 $13.2 \%$, 测量装置如图 23 所示。

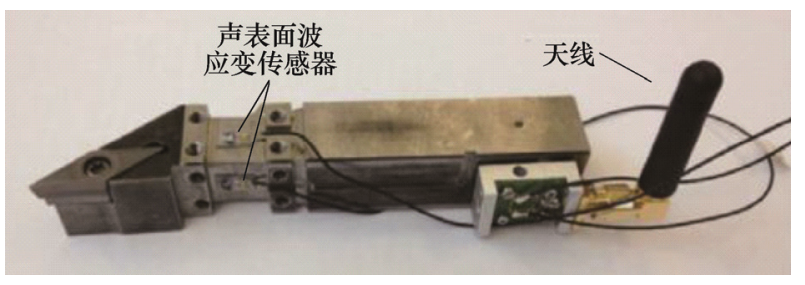

图 23 双向声表面波测力刀具 ${ }^{[46]}$

可以发现，利用声表面波实现对切削力测量的 主体测量原理依然是基于刀具受力后所产生的应变 及其后续影响，所采用的信号处理方式稍显复杂， 提出的刀具结构与应变式测力刀具结构类似, 并 且对于切削力的解耦研究仍有许多工作要做。但 作为一种新兴的切削力测量刀具, 丰富了智能刀 具的测量方式, 为智能刀具研究注入了新理念及创 新思路, 随着学者不断地深入研究, 此种测量方式 会日渐成熟。

\section{2 切削温度自感知刀具}

在切削加工过程中, 切削温度是影响加工质量 的重要因素之一, 切削温度主要是由刀具与工件间 的摩擦以及切削层发生弹塑性变形所引起, 切削温 度的升高会导致刀具磨损加剧, 降低刀具寿命, 致 使被加工件质量难以保证。采用切削温度在线测量 技术可以实时测量切削温度变化, 对提升刀具寿命 具有重要意义, 同时为优化控制切削状态提供了重 要的状态参数 ${ }^{[47-48]}$ 。以下对切削温度测量技术进行 具体论述。

SUGITA 等 ${ }^{[49]}$ 提出在刀具前刀面集成微型阵列 传感器的测温刀具, 如图 24 所示。通过激光对刀具 前刀面进行刻蚀, 随后涂覆绝缘膜与铬膜, 利用刀 具本身作为热电偶一极形成硬质合金(WC-Co)-铬
(Cr)热电偶, 该传感器对温度的测量可实现 $10 \mathrm{~ms}$ 的快速响应, 由于传感器的小型化可在刀具前刀面 布置多个传感器。

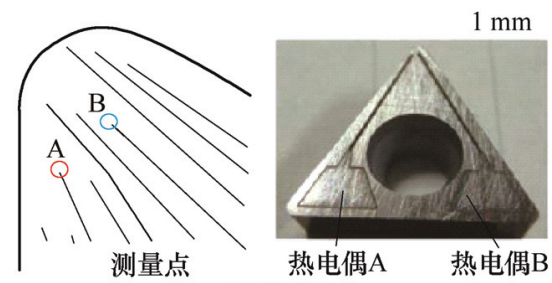

(a) 测量点

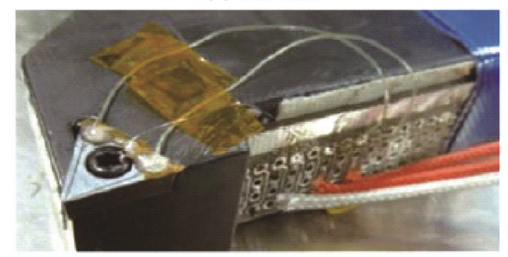

(b) 刀具整体结构

图 24 传感器及系统安装[49]

崔云先等 ${ }^{\left[{ }^{[0]}\right.}$ 提出了一种基于 $\mathrm{NiCr}-\mathrm{NiSi}$ 薄膜热 电偶的瞬态切削温度测量刀具, 热电偶布局如图 25 所示, 采用直流脉冲磁控溅射技术将 NiCr-NiSi 薄 膜热电偶嵌入到刀具后刀面刀尖位置, 热电偶传感 器直接参与切削并跟随刀具一起磨损。试验结果表 明, 所研制的测温刀片在 $30 \sim 300{ }^{\circ} \mathrm{C}$ 范围内具有良 好的线性，最大线性误差不超过 $0.92 \%$, 所研制的 智能测温刀具可以快速准确监测 $0.1 \mathrm{~s}$ 内刀具刀尖 处瞬态切削温度的变化。

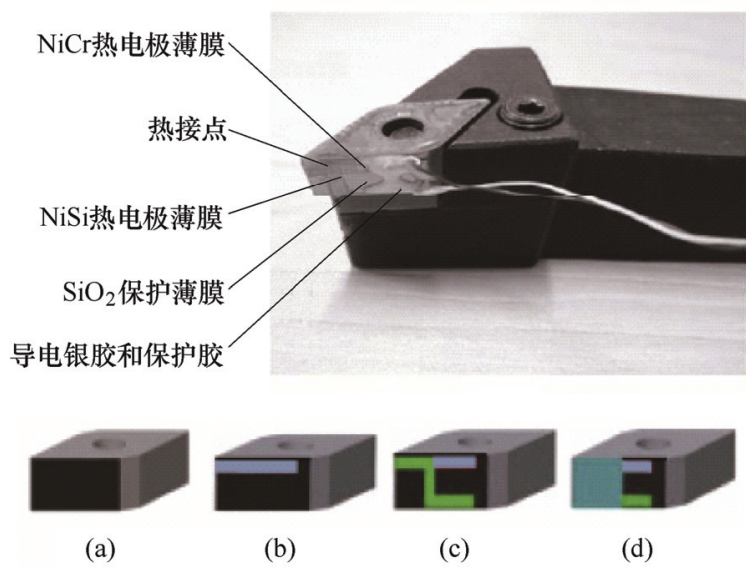

图 25 瞬态切削温度测量刀具 ${ }^{[50]}$

李林文等 ${ }^{[51}$ 通过在 $\mathrm{PCBN}$ 刀具嵌入微尺度薄膜 热电偶阵列的方法对硬态切削过程中切削温度的变 化进行测量, 如图 26 所示。10 组热电偶测试点被 安装于刀尖位置处, 测试点沿着前后刀面的边界排 布, 所有测试点所占的安装总面积不到 $1 \mathrm{~mm}^{2}$ 。通 过试验证明采用所提出的热电偶测温方法很好地实 现了刀具温度的测量。 


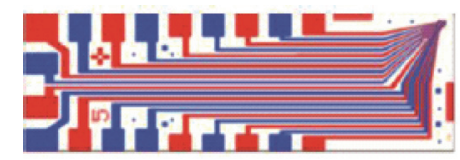

(a)

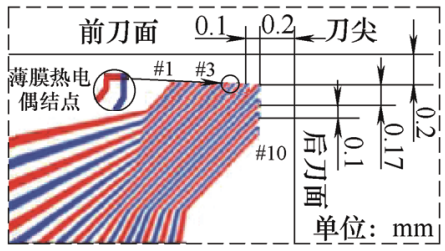

(c)

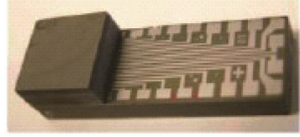

(b)

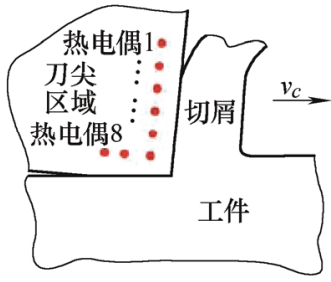

(d)
图 $26 \mathrm{PCBN}$ 刀片和薄膜热电偶阵列布局 ${ }^{[51]}$

陶波团队 ${ }^{[52]}$ 利用刀具表面微织构实现薄膜传 感器在硬质合金刀具刀刃区域进行集成制造的方 法, 提出了集成薄膜热电偶阵列传感器的硬质合金 智能刀具, 如图 27 所示。解决了传感器无线供电与 数据同步传输, 实现了难加工材料高速切削过程中 刀具近切削区域温度的高精度跟随测量。

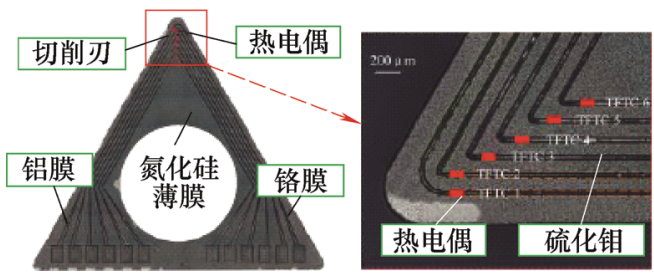

图 27 薄膜热电偶阵列测温刀具 ${ }^{[52]}$

舒盛荣等 ${ }^{[53]}$ 提出一种用于对切削温度进行测 量和调控的智能刀具, 具体结构如图 28 所示。在硬 质合金刀片下设置一个冷却通道，在加工过程中通 过冷却液的循环流动将热量带走, 并利用热电偶对 进出口冷却液温度进行实时测量, 进而推算出刀尖 处的温度, 该刀具的使用能够有效地降低刀具温度, 并实现了对刀具切削温度的测量。

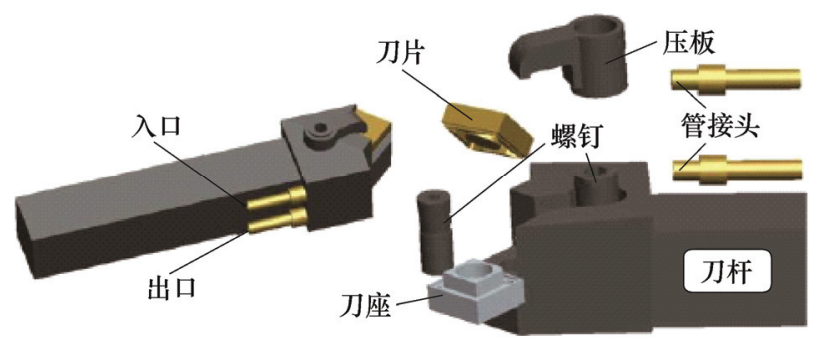

图 28 切削温度测量与调控刀具 ${ }^{[53]}$

$\mathrm{COZ}$ 等 ${ }^{[54]}$ 提出了一种旋转式刀具温度测量系 统, 实现对钻头、端铣刀切削温度的测量, 应用于 钻头的热电偶布局如图 29 所示。在钻头与端铣刀切 削刃附近预埋热电偶, 并在专用刀架上集成数据采 集系统和无线发射单元对信号进行采集、传输, 实
现了旋转刀具切削刃周围温度的有效测量。

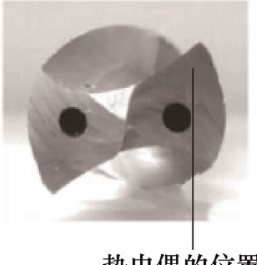

热电偶的位置
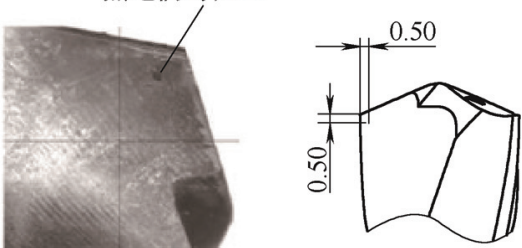

图 29 钻头热电偶布局 ${ }^{[54]}$

WRIGHT 等 ${ }^{[55]}$ 研制了一种用于铣削刀具温度 测量的无线测温刀具, 电阻式温度传感器安装在端 铣刀的背面, 通过补偿导线连接至刀柄中的无线平 台, 实现信号的传输, 如图 30 所示。该温度刀具可 作为开放式数控机床的感知部分集成于刀杆中，实 现切削过程中温度的监控。

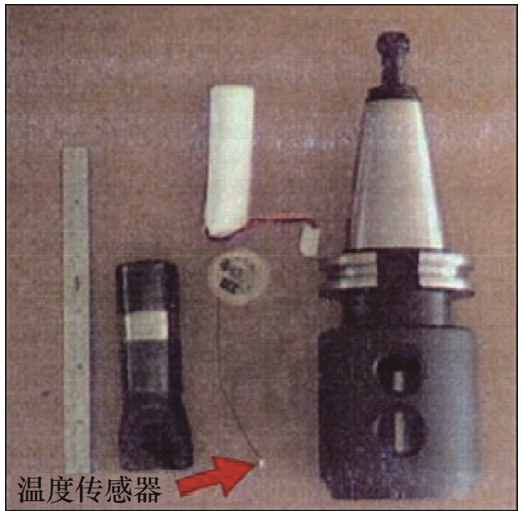

图 30 无线式铣削测温刀具 ${ }^{[55]}$

KERRIGAN 等 ${ }^{[56]}$ 研发出一种应用于碳纤维复 合材料加工的智能无线测温刀具, 如图 31 所示。将 直径为 $0.2 \mathrm{~mm}$ 的 $\mathrm{K}$ 型热电偶精确地嵌入 $0.6 \mathrm{~mm}$ 的 刀具凹槽中, 保证热电偶距切削刃底部 $0.5 \mathrm{~mm}$ 的距 离。实现了碳纤维复合材料铣削加工时切削温度的 测量。

现有切削测温刀具多是将热电偶或薄膜传感器 嵌入或粘贴在刀具切削刃附近，实现对刀具切削区 域温度的拾取及测量，采用有线或无线的信号传输 方式对数据进行采集。测温刀具的技术难点主要包 括以下几方面: (1) 热电偶传感器存在测量瞬态温度 响应速度不足问题; (2) 将测温传感器设置在切削区 域易产生磨损及破坏，影响测量精度; (3) 实现测温 功能多数需要对刀片进行特殊处理, 制作过程相对 复杂, 可以满足试验室研究需求, 但实现工程应用 


\section{存在较大难度。}

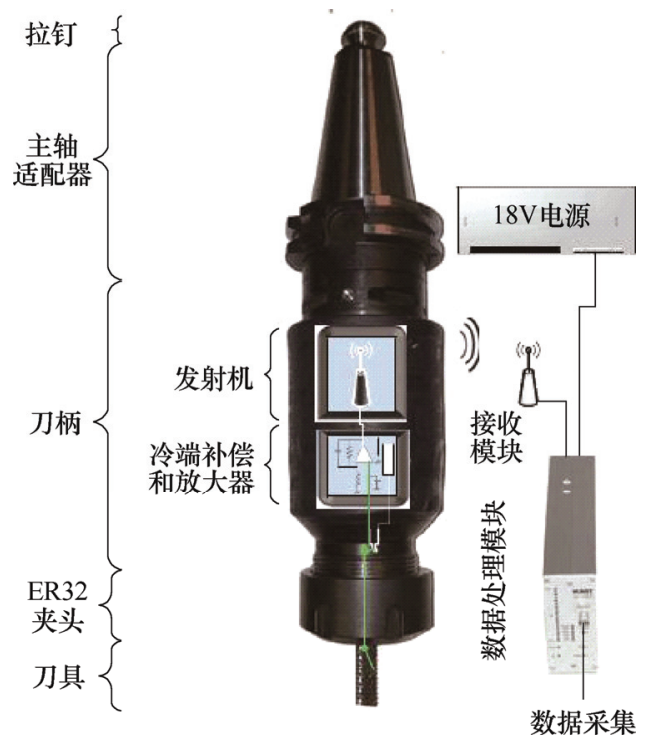

图 31 碳纤维智能无线测温刀具 ${ }^{[56]}$

\section{3 切削振动自感知刀具}

振动始终伴随着切削加工, 切削振动的发生会 影响被加工件的形状精度和表面质量, 同时对机床 精度及操作环境造成影响, 振动严重时甚至会发生 刀具破损, 导致工件报废。通过监测切削振动的状 态来判断加工状态在实际生产中已被广泛采用, 学 者们也提出了多种具备振动测量功能的刀具。

DEVILLEZ 等 ${ }^{[57]}$ 利用电泞流位移传感器对刀 具加工过程中的振动位移进行测量, 结构如图 32 所示。在车刀端部安装一个附加质量块, 电浴流传 感器布置在附加质量块后方, 通过测量附加质量的 振动位移, 进而得到刀具的振动位移, 实现了传感 器远离刀具的振动测量。

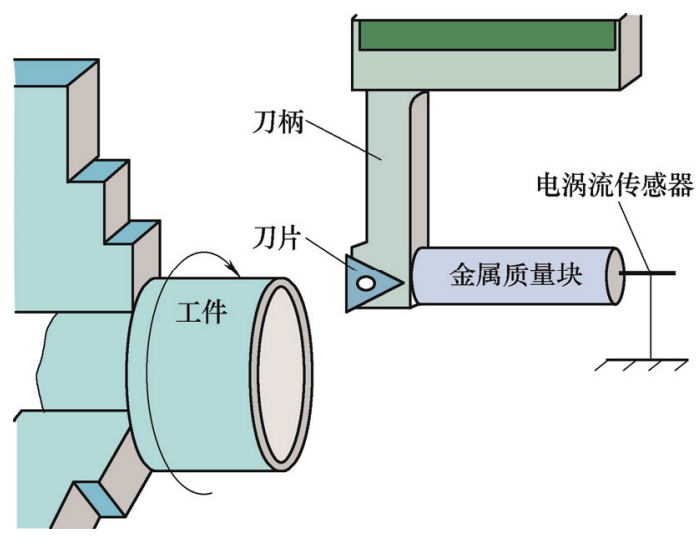

图 32 车削振动位移测量刀具 ${ }^{[57]}$

CHUNG 等 ${ }^{[58-59]}$ 为降低测量系统对切削加工过 程的侵入性, 解决有线式传感器带来的安装不便问 题, 提出基于电磁感应的自发电切削振动测量系统, 如图 33 所示。在刀具主轴部分布置 3 个加速度传感
器, 在刀柄圆周上固定 4 个永磁铁, 外侧布置感应 线圈装置, 当铣削时刀柄旋转时线圈中感应出电流, 为振动测量系统提供电能, 从而实现振动信号监测, 整个系统功耗为 $223.35 \mathrm{~mW}$, 需要主轴转速在 $1650 \mathrm{r} / \mathrm{min}$ 以上才能使振动测量系统正常工作。

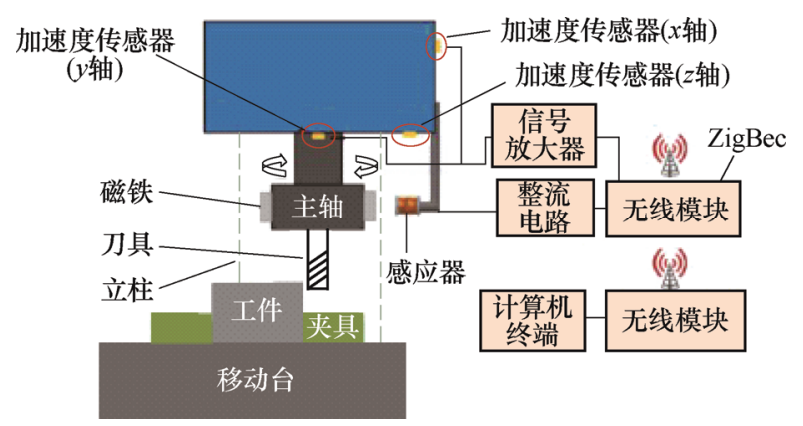

图 33 自发电切削振动测量系统 ${ }^{[58]}$

PENG 等 ${ }^{[60]}$ 研制了一种镗杆径向微位移两点式 测量刀具系统, 系统结构如图 34 所示。为了精确 加工非圆柱销孔, 设计了一种高性能超磁致伸缩 致动器智能镗杆, 通过控制通入线圈电流, 可精 确控制镗杆旋转角度和偏移位置, 为了能够实时 测量出镗杆偏移量, 在系统中集成两个正交布置 的电浴流位移传感器和一个角度编码器, 可在线 测量出镗杆微位移的变化量, 通过数学模型的建 立和误差分析, 可精确测量出径向微位移, 且算 法简单、效率高。

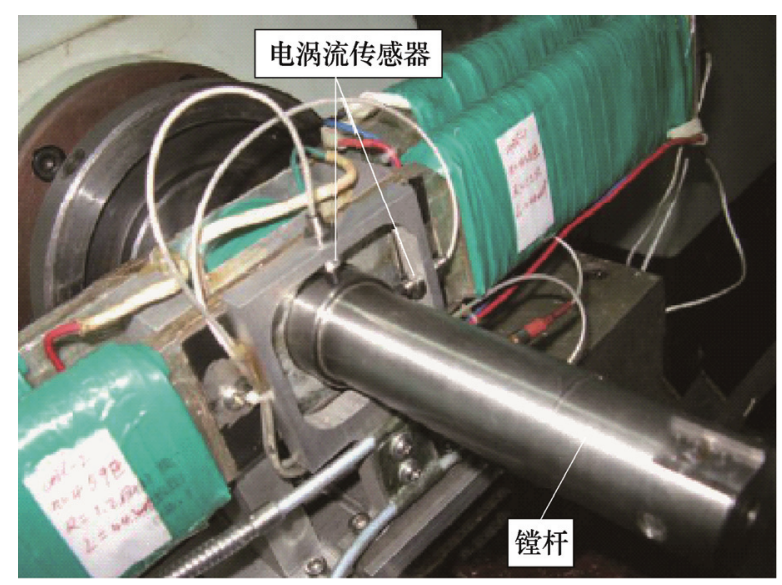

图 34 两点式微位移测量刀具系统 ${ }^{[60]}$

SUPROCK 等 ${ }^{[61]}$ 为了能够精确地观察数控金属 切削系统的动力学特性, 研制了一种集成到商用立 铣刀刀柄的低成本无线振动监测系统, 该系统采用 驻极体电容器作为加速度传感器, 通过电容模块阻 抗的改变来实现振动监测。结构如图 35 所示, 加速 度传感器安装在端铣刀刀片背面, 通过引线连接到 信号采集传输平台上, 采用无线蓝牙信号传输方式, 该系统具有对切向振动反应灵敏的优点。 


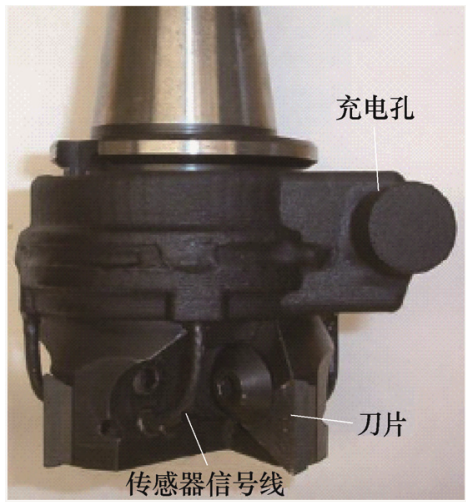

图 35 无线振动监测系统 ${ }^{[61]}$

刘海军等 ${ }^{[62]}$ 提出了将振动传感器以及信号采 集电路集成到刀柄上的智能刀具, 采用 BT50 刀 柄为原型, 将传感器与刀柄进行集成, 具体结构 如图 36 所示。通过分析振动产生原理, 建立了速度 反馈型振动和位移反馈型振动两种模型, 并对系统 进行了整机测试, 结果显示噪声信号干扰很小, 系 统所测得的振动信号比在工件上测得的信号强度 高, 确定此方案可较为有效地对振动信号进行测量。

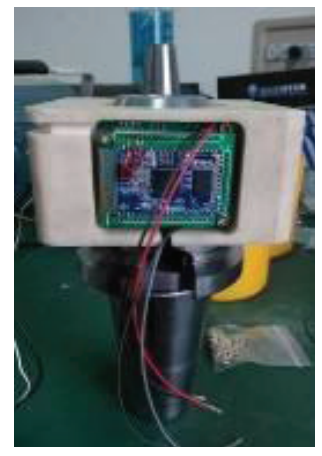

图 36 集成化振动测量刀具 ${ }^{[62]}$

由于刀具的振动可较好地反映实际加工情况, 通过监测振动信号对加工状态进行判断已经越来越 成为较为普遍的方法。目前在实际切削加工中多采 用安装商用传感器的方式实现对切削振动的测量, 同时, 具有振动测量功能的集成化刀具也在不断被 开发, 随着智能刀具技术研究不断完善, 一体化的 振动测量刀具会大量出现。

\section{3 面向切削过程的智能刀具控制技术}

对于切削过程中的控制, 通常采用控制切削参 数(转速、进给、切深)来实现, 此种操作是在机床 设备端实现的。采用智能刀具同样可实现对切削过 程的控制, 此种方式是在刀具端完成的, 通过对智 能刀具自身特殊结构相关参数的调节, 可实现对切 削过程的控制。

\section{1 切削过程中切削力控制研究}

切削力是加工中的重要状态信息, 切削力的变 化可较为直观地反映切削过程的状态变化, 通过对 切削力的控制可实现对加工质量及效率的控制。在 加工中对切削力的控制具有重要意义，可避免局部 切削力过大、崩刃或断刀情况的发生。可实现平稳 切削, 减少加工中的振动, 降低主轴所受冲击等。 采用智能刀具的特殊结构及控制算法对切削力进行 在线调控已成为智能刀具研究的重要课题。

GRANADOS 等 ${ }^{\left[{ }^{[} 3\right]}$ 应用常规切削力控制系统研 制了一种非刚性微尺度切削系统, 如图 37 所示。在 柔性悬臂梁上安装单晶金刚石刀具，采用电容式位 移传感器测量柔性悬臂梁的变形, 通过反馈控制系 统和压电驱动器保持位移传感器与测量板之间的相 对位置不变, 最终依靠位移传感器、反馈控制系统 和压电驱动器的协同工作实现对切削力的调控, 从 而在切削过程中保持恒定的切削力。

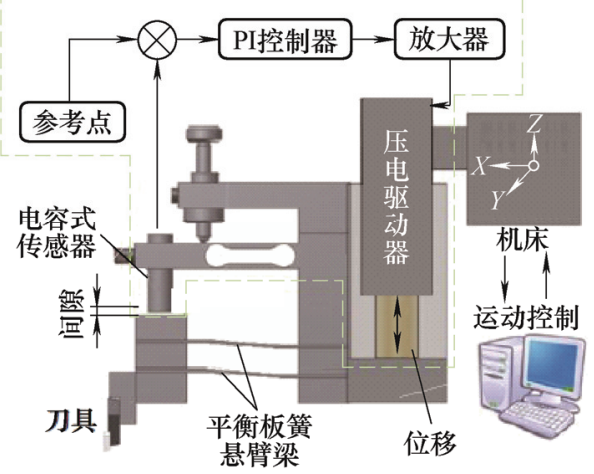

图 37 非刚性微尺度切削系统 ${ }^{[63]}$

LI 等 ${ }^{[64-65]}$ 将柔性机构与精密工程相结合, 提出 一种新型快速刀具伺服系统, 如图 38 所示。压电执 行器与位移传感器安装于刀具后端, 通过伺服系统 可实现刀具沿 $Z$ 向移动。压电执行器驱动电压为 30 $\mathrm{V}$, 行程长度为 $3.829 \mu \mathrm{m}$, 快速刀具伺服系统静刚 度为 $10.88 \mathrm{~N} / \mu \mathrm{m}$, 最终实现使用金刚石切削刀具对 微特征或自由形状表面进行精密车削。

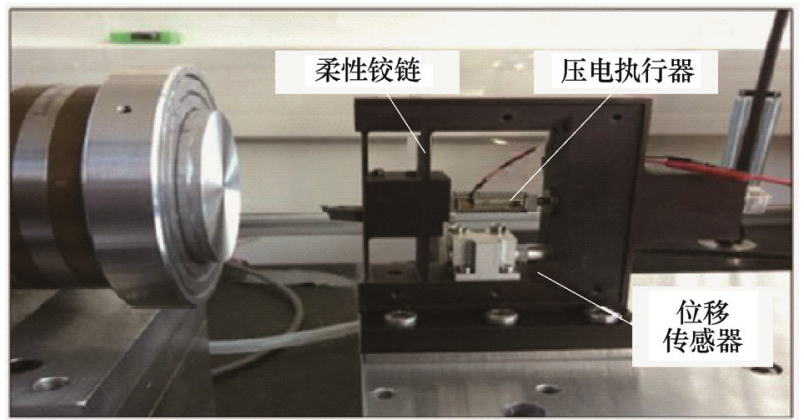

图 38 快速刀具伺服系统 ${ }^{[65]}$ 


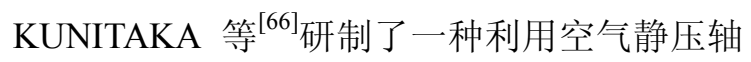
承实现对硬脆材料进行恒力切削的刀具系统, 通过 调节供气压力实现对切削力的调节。如图 39 所示, 恒力切削系统中设置三组空气静压轴承, 通过对三 组空气静压轴承气压的控制实现对切削力的调控, 从而实现刀具在切削过程中始终保持恒定的切削 深度。

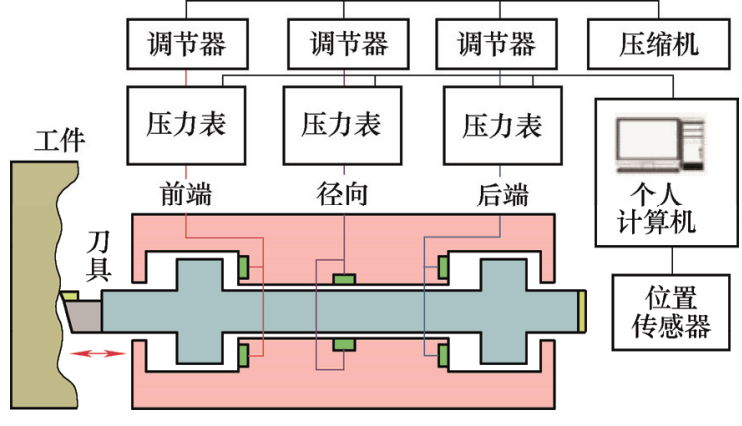

图 39 恒压装置示意图 ${ }^{[66]}$

通过对以上切削力控制刀具分析可以发现, 涉 及切削力控制的智能刀具多数为车削刀具, 主要原 因在于相比于铣削而言, 车削刀具的切削力控制结 构更容易实现, 而对于几何特征较为复杂的曲面 铣削加工, 在刀具侧实现切削力控制具有一定难 度。对于特定的加工场景, 采用切削力控制刀具 必然会在加工质量与效率提升方面表现出优异的 切削性能。对于切削力控制刀具依然需要在刀具 结构、驱动器、控制算法方面开展大量且深入的 研究, 并加大铣削刀具研究, 结合具体工况扩大 切削力控制刀具的应用范围, 探索切削力控制加 工工艺。

\section{2 切削过程中温度控制研究}

在切削过程中, 切削温度对工件表面质量的影 响较大, 过高的切削温度会致使零件表面烧伤, 同 时较高的切削温度会导致刀具磨损加剧, 降低刀具 寿命, 无论是为了提高刀具寿命和生产效率, 还是 为了安全、高可靠性的生产, 金属切削过程中都应 对切削温度进行有效控制。

舒盛荣 ${ }^{[67]}$ 提出了一种内冷式智能刀具系统, 如 图 40 所示。在刀垫上设置冷却槽, 冷却槽与刀垫内 布置的冷却液通道相连接, 采用蒸馏水作为冷却液, 通过冷却液的循环流动将刀片的热量带走。建立了 内冷式智能车刀热特性解析理论模型, 获得了切削 平均温度、出口温度的时间响应解析表达式, 实现 了对切削温度的实时监测。在切削过程中通过对冷 却液流量的控制, 降低切削温度, 从而减缓刀具在 高切削温度下的磨损速率。

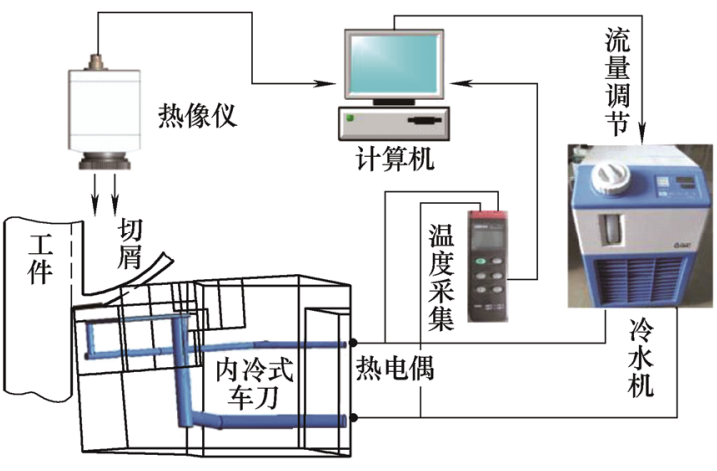

图 40 内冷式智能刀具系统 ${ }^{[67]}$

刘文博等 ${ }^{[68]}$ 提出内冷式智能车刀温度控制系 统, 冷却液从车刀内部的管道通过, 采用热电偶对 冷却液进口与出口的温度进行测量, 当冷却液出口 温度超出设定阈值时, 通过增加变量洜的流量对刀 具的切削温度进行调节, 具体结构如图 41 所示。系 统集成了内冷车刀温度信号采集、数据显示、温度 报警功能, 实现了对车刀温度的有效监控与调节。

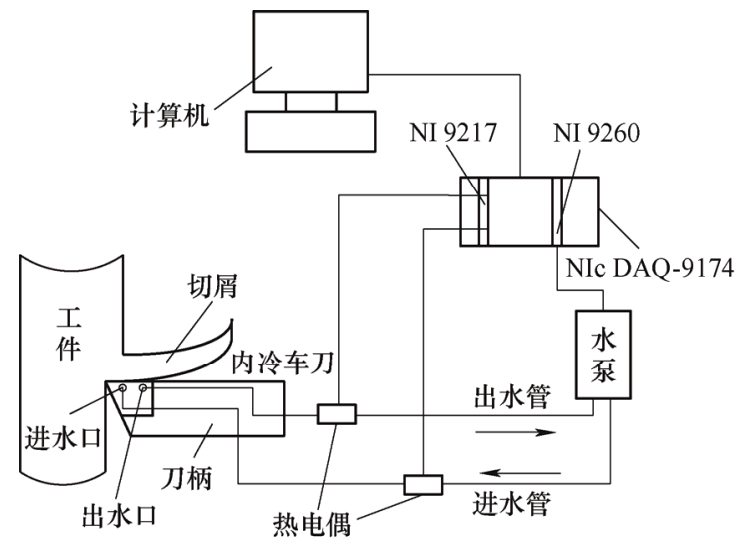

图 41 内冷式智能车刀温度控制系统 ${ }^{68]}$

SUN 等 ${ }^{[69]}$ 同样提出了一种用于干式切削的内冷 式智能刀具, 在刀尖附近设计了一个简单多变的内 部冷却结构, 如图 42 所示。该刀具通过连续测量冷 却液进、出口温度, 通过智能算法进行关联, 实时 获得刀具尖端温度, 机床能够根据切削温度自适应 地进行优化加工, 即调整加工过程切削参数和进口 流量, 以实现加工中切削温度的控制。

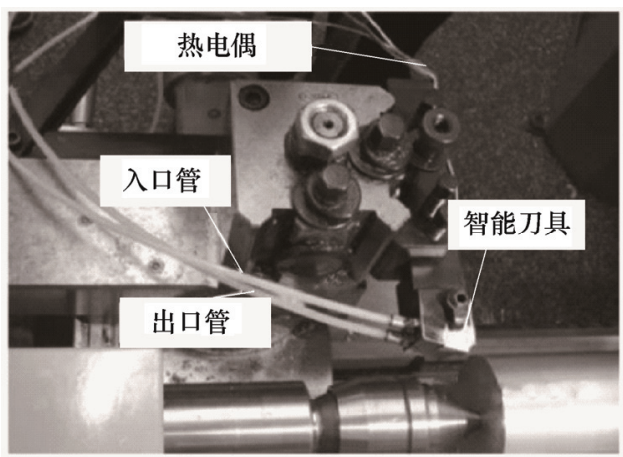

图 42 干式切削内冷式智能刀具 ${ }^{[69]}$ 
梁良等 ${ }^{[70-71]}$ 提出了基于热管散热的车削刀具, 系统组成如图 43 所示。在刀片底部加工盲孔用于嵌 入热管的蒸发端, 在对热管刀具上特定点及刀-屑接 触面温度测量试验的基础上, 建立了热管刀具的三 维传热模型, 并采用传热反求方法对热管刀具在不 同切削参数条件下刀-屑接触面上的热流密度及刀屑接触面温度进行优化求解, 采用热管刀具可以导 出刀具中 $28 \% \sim 42 \%$ 的热量。

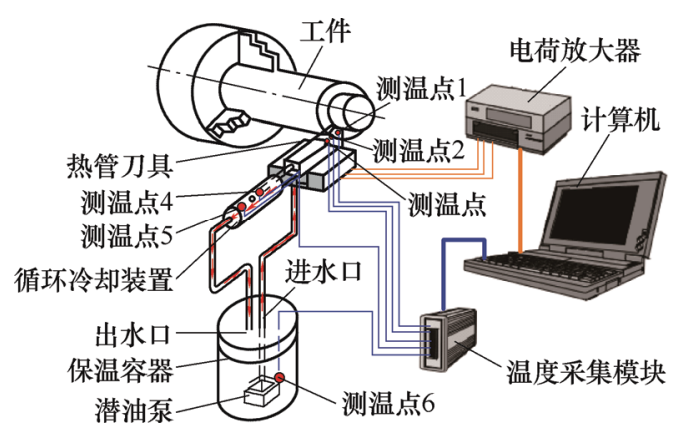

图 43 热管散热式车削刀具 ${ }^{[71]}$

对于刀具切削温度控制的研究内容多集中在车 削刀具, 原因在于车削刀具前刀面的热量由于切 屑的覆盖不易散出, 是加工中关注的交点。同时, 由于车削刀具的结构特征更容易实现对冷却结构 的布置。而铣削刀具在不参切削时, 通过自身旋 转所产生的风冷作用可以散失部分热量。现有智 能控温刀具在冷却结构、温度测量、智能控温算 法、切削工艺控制等方面依然需要开展大量而深入 的研究工作。

\section{3 切削过程中振动控制研究}

切削过程中总是伴随有振动现象, 振动的发生 会降低加工质量、使工件表面产生振纹、加快刀具 磨损和破损, 影响机床精度。学者们提出了不同结 构及原理的智能刀具对切削过程中的振动进行 抑制。

$\mathrm{LU}$ 等 ${ }^{[72]}$ 研制了内部安装光纤位移传感器的非 接触式磁力驱动器, 并将其应用于镗杆的减振。该 驱动器由四个相同的线性化电磁单元构成, 所产生 的磁力可以在两个径向方向对镗杆进行驱动, 显著 提高了刀具系统的动态刚度, 结构如图 44 所示。

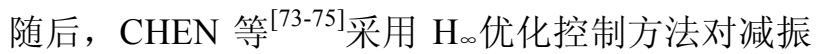
镗杆进行控制, 大大增加了镗杆的稳定性和材料去 除率。

刘强等提出一种内置变刚度动力吸振器的智能 减振镗杆, 镗杆结构如图 45 所示, 将变刚度动力吸 振器安装在镗杆前端的空腔内, 通过调整伸缩式悬 臂梁的长度实现对动力吸振器刚度的调节, 结合对 刀具系统动力学模型分析, 实现对智能减振镗杆减
振性能的最优调节 ${ }^{[76]}$ 。

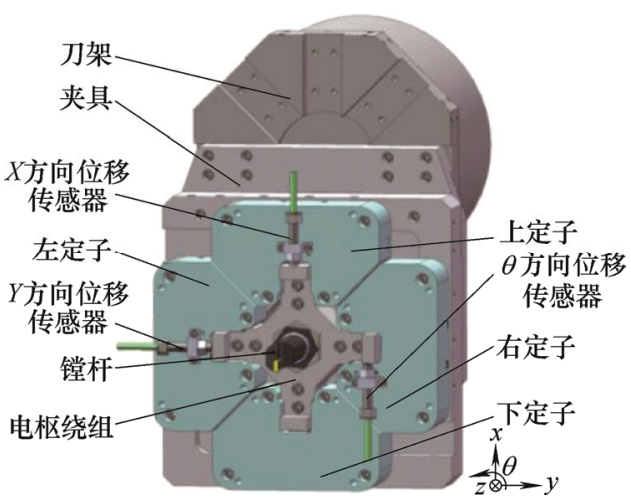

图 44 磁力驱动式减振镗杆 ${ }^{[72]}$

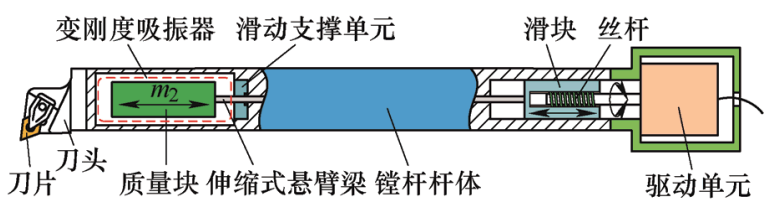

图 45 变刚度动力吸振式减振镗杆 ${ }^{[76]}$

MATSUBARA 等 ${ }^{[77]}$ 通过在镗杆杆体上安装压 电驱动器来抑制镗杆的振动, 具体结构如图 46 所 示, 压电驱动器连接在电感电阻(LR)的电路中, 为 了提供足够的力和刚度, 在镗杆上设计了叠层式压 电驱动器, 并在车床上采用优化调整后的 LR 电路 对减振镗杆动态特性进行评价, 试验结果表明所设 计的减振镗杆成功地实现了减振。

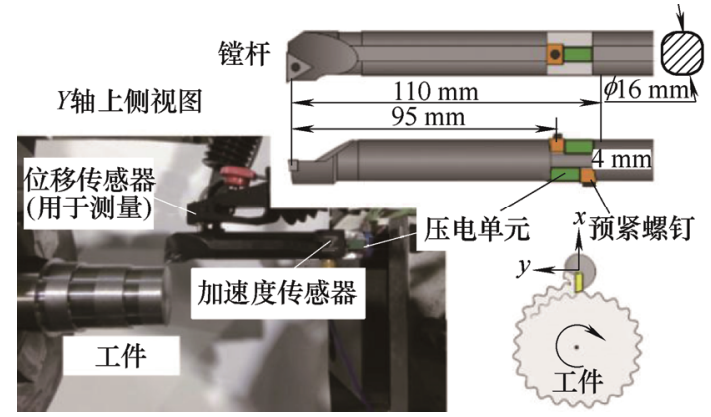

图 46 减振镗杆及试验装置 ${ }^{[77]}$

王民等 ${ }^{[78-79]}$ 利用电流变材料的电控流变特性, 设计一种调控杆体动态特性的减振镗杆, 减振镗杆 的结构如图 47 所示, 杆体与支撑套之间填充电流变 液, 通过调节电场的强度控制电流变液的特性, 电 流变液在系统中具有屈服前和屈服后两种变形模 式，在屈服前相当于黏性元件和弹性元件的并联， 屈服后相当于黏性元件和摩擦阻尼元件的并联。在 不同的加工情况下选择最佳的电场强度控制屈服 力, 使系统刚度对切削振动微小变化产生灵敏反应, 使动态切削力激发不了镗杆共振。 
正 电流绝缘

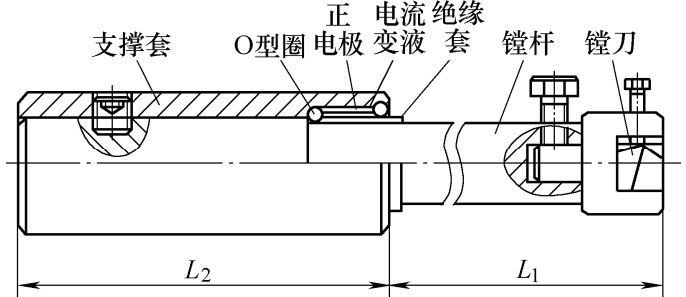

图 47 电流变液减振镗杆 ${ }^{[79]}$

梅德庆等 ${ }^{[80-81]}$ 提出了一种基于磁流变液的智能 减振镗杆, 通过改变所施加磁场的强度对系统刚度 进行调节, 从而实现对颤振的抑制, 镗杆的体性能 测试现场如图 48 所示。通过理论分析和数值模拟均 表明, 大振幅、中等频率的方波励磁电流对再生颤 振抑制效果较好。试验结果表明, 在频率为 $4 \sim 6 \mathrm{~Hz}$ 、 振幅为 $0 \sim 2 \mathrm{~A}$ 的方波激励电流作用下, 镗杆的颤振 能够得到显著抑制。孔天荣等 ${ }^{[82-84]}$ 对磁流变减振镗 杆的切削颤振抑制机理进行了研究, 李欣 等 ${ }^{[85-86]}$ 基于经验模态分解和希尔伯特-黄变换对磁流变自 抑振智能镗杆颤振在线预报理论和方法进行研究。

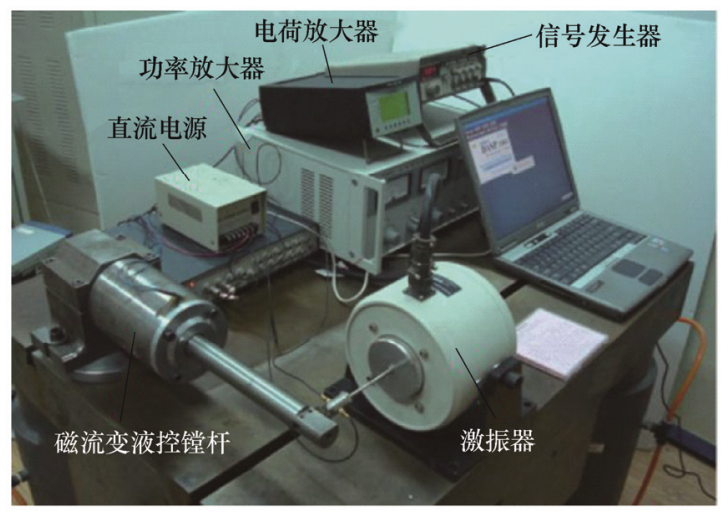

图 48 磁流变液减振镗杆性能测试现场 ${ }^{[81]}$

MONNIN 等 ${ }^{[87]}$ 提出了一种集主动控制系统与 高性能电主轴于一体的智能刀具振动控制系统, 如 图 49 所示。主动控制系统由推拉式工作的两对压电 堆叠执行器组成, 并安装于电主轴前端的轴承支承 处, 可实现对电主轴径向位移的控制。同时在前轴 承处安装了两个垂直定位的加速度传感器用于测量 轴承支架的径向振动。在建立了结构动力学模型基 础上, 提出了干扰抑振策略和稳定抑振策略, 并对 两种策略的优缺点进行了分析。

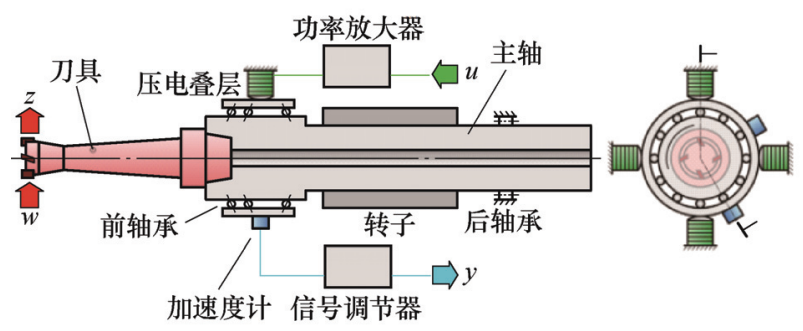

图 49 智能刀具振动控制系统 ${ }^{[87]}$
以上分析可以发现，针对振动控制的智能刀具 研究成果较为丰富, 采用的刀具振动控制原理较多, 刀具抑振结构各有不同，涉及多种智能材料的应用 与控制。目前, 振动控制智能刀具在抑振结构、智 能材料使用及振动控制方法的研究较多, 将振动状 态监测与振动控制相结合, 实现刀具振动状态的自 感知及智能控制研究较少, 对此部分研究内容应开 展深入研究。

\section{4 智能刀具关键技术}

智能刀具作为智能切削加工的执行端, 已成为 未来刀具的重要发展方向, 涉及多学科的交叉研究, 具体的关键技术包括: (1) 切削状态监测技术; (2) 刀 具切削性能调控技术; (3) 结合互联网与大数据的智 能学习算法。

\section{1 切削状态监测技术}

切削过程中对于切削状态的监测是确保刀具进 行有效自我调控的基础, 学者们对刀具切削状态的 监测研究较早, 取得了大量的研究成果。

(1) 传感器技术。

将传感器集成在刀具结构中从而对切削状态进 行监测是较为常规的智能监测刀具研究手段, 需要 解决的主要问题包括传感器的布置安装、刀具结构 强度的保证、信号采集、系统供电等。如何实现传 感器与刀具结构的优化集成, 在最大限度不影响刀 具切削性能的前提下实现传感器在刀具内部的有效 嵌入, 优化传感器的布局及刀具安装结构是重要的 研究内容。随着传感器技术的发展, 响应速度快、 精度高、性能稳定的传感器在智能刀具中的应用必 定会使智能刀具在状态监测的准确性和实时性方面 实现较大的性能提升。

（2）精巧的刀具状态监测结构。

传感器在刀具上的安装布局需要进行优化设 计，在保证刀具结构自身强度的基础上，如何利用 刀具自身结构特点, 提出结构紧凑、精巧的刀具结 构, 以方便传感器的安装、固定具有重要意义。另 一方向, 可通过对刀具自身结构进行精巧设计, 使 刀具自身结构作为感知单元, 感知切削过程状态变 化, 利用精巧结构使刀具同时具备切削与感知功能。 如何对刀具结构进行模块化、集成化、优化设计是 一项重要的研究内容。

(3) 信号采集及特征提取。

在状态监测中, 针对不同的切削状态监测需求, 所采用传感器的感知原理各不相同, 由于智能材料 
的不断涌现及现有智能材料的不断更新, 对于信号 采集算法的研究始终是一项重要研究工作, 如何提 出采集算法简单、运算速度快、准确率高、采集结 果稳定的信号采集算法是研究重点。此外, 传感器 的安装位置及布局直接对监测信号的数值产生影 响, 传感器的安装位置与所测量物理量之间的关系 模型建立显得尤为重要, 对所监测的物理量进行状 态解耦研究同样需要给予足够的关注。

在此基础上对采集的信号进行特征提取, 涉及 多种信号处理方, 实现信号的压缩、降维、特征提 取, 从而实现刀具真实物理状态的监测。对于信号 特征提取的研究关注点集中在特征提取算法的准确 性、实时性、稳定性、计算速度等。

(4) 多状态监测。

学者们对于切削状态监测刀具的研究已取得较 多研究成果, 提出了多种切削状态自监测刀具, 监 测的状态集中在切削力、刀具振动和切削温度。但 大多数切削状态监测刀具多是对单一的切削状态进 行监测, 同时对切削过程中多种物理量进行监测的 智能刀具研究较少, 需对集成化、模块化的多状态 智能监测刀具开展深入研究, 实现在一把刀具多种 切削状态的同时监测。

\section{2 刀具切削性能调控技术}

(1) 刀具性能调控系统。

智能刀具自身性能的调节依靠刀具内部或外置 的调控系统来实现, 涉及机、电、液、气及软、硬 件的协同工作。例如, 对于切削温度的调控, 需要 以蒸馏水或液氮等作为冷却介质, 冷却系统通过对 冷却介质流量的调节实现对切削温度的调节。对于 切削力的调节需要调控系统对压电执行器、磁力驱 动等驱动装置进行调节, 从而实现对切削力的调控。 对振动的调节同样会应用智能材料作为执行器以及 动力吸振器、摩擦吸振器、电/磁流变液等振动调控 装置, 通过调控系统对振动调控装置的调节实现对 刀具减振性能的调节。

智能刀具涉及的调控装置多样, 驱动元件多样, 调控原理各不相同, 对于驱动元件、驱动结构、调 控原理等方面都需要开展大量的研究工作, 对驱动 系统及结构进行优化, 增加驱动元件与刀具的集成 性, 提出结构紧凑、响应速度快、动作准确的调控 系统。同时要减小调控系统硬件体积, 增加硬件的 集成化、模块化程度, 使调控系统具有较高的通用 性和适用性。

(2) 调控算法。

由于智能刀具对与切削力、振动、切削温度等
的调控装置结构及原理各不相同, 需要针对刀具的 具体调控结构及调控的物理量制定相应的调控算 法。这就导致对于智能刀具的调控算法多样, 不 同刀具所采用的调控算法各不相同, 研究人员需 要在深入分析刀具结构及调控原理的基础上, 根 据具体调控需求及驱动结构的特点提出定制化的 调控算法, 并对控制算法进行优化迭代。具体需 要在调控算法的控制准确性、稳定性、实时性等方 面开展研究。

\section{3 结合互联网与大数据的智能学习算法}

伴随工业互联网、大数据、云计算、人工智能 的快速发展, 将互联网、大数据、人工智能技术应 用于智能刀具已成为智能刀具研究内容的重要部 分, 为智能刀具的研究注入了新的思路, 需要开展 较为深入的研究。

现有智能刀具的工作方式多是通过刀具自身或 外部的监测设备获得刀具的切削状态信息, 调控系 统根据所获取的状态信息及建立好的控制程序对智 能刀具进行性能调控, 从而实现对加工过程的有效 控制。此种方式只是对所获取的状态数据进行了在 线处理, 并没有实现对数据的储存、共享及依托人 工智能的数据分析与挖掘, 现有智能刀具的自动化 程度较为完备, 但智能不足, 是弱智能刀具。

智能刀具的研究需进一步依托工业互联网技术 实现对监测数据的快速存储与共享, 同时借助于大 数据分析和深度学习等人工智能手段, 可对切削过 程的历史监测数据进行整合分析, 将状态监测信息 与历史数据相结合, 建立刀具状态监测信号与切削 性能的映射关系模型, 进而通过数据学习使刀具变 得更加 “聪明”, 实现刀具性能的智能化调控, 使智 能刀具具有强智能。

\section{5 结论与展望}

本文对智能刀具进行了介绍, 探讨了智能刀具 在切削状态监测与切削过程控制的最新研究进展, 并对智能刀具的关键技术进行了论述, 主要结论及 展望如下。

(1) 智能加工技术是加工技术的必然发展方 向, 智能刀具作为加工中的工具终端, 是智能加工 中的重要一环, 对智能刀具开展深入的理论及应用 研究是智能制造的必然需求, 在涉及重大装备核心 零部件加工、特种加工及批量化生产中, 智能刀具 必然会替代传统刀具发挥重要作用, 对于智能刀具 应开展广泛而深入的研究, 大力开发智能刀具的使 
用范围及应用场景, 实现智能刀具工业化应用。

(2) “感知型” 智能刀具的研究较为深入, 成果 丰富, 实现了对切削力、切削温度、刀具振动等的 在线监测, 所采用的监测原理多样, 使用的传感器 种类各不相同, 对于不同的监测需求提出了多种状 态监测算法。目前, 智能刀具对于单一的切削状态 监测技术已相对成熟, 但对于多状态监测研究还需 要开展大量工作, 如何在一把智能刀具上对多种传 感器进行融合与集成, 实现切削状态多物理量的同 时监测是智能刀具状态监测的研究重点。

(3) 智能刀具研究属于多学科交叉研究, 涉及 切削机理、传感器技术、测试技术、数据处理、智 能控制、互联网、大数据、云计算、人工智能等多 个领域, 伴随所涉及领域技术的不断发展, 任一领 域中的技术取得突破都会对促进智能刀具技术的提 升。同时, 也需要看到智能刀具系统的复杂性, 由 于涉及领域众多, 研究人员需具备多学科交叉融合 的研究能力, 围绕智能刀具开展各领域间的协同合 作, 从而可推动智能刀具技术的快速发展, 实现关 键技术的多路开花, 多路结果。

(4) 智能刀具的研究在实验室层面已取得一定 的成果, 在一定程度上已实现了改善加工过程, 提 升加工质量、效率的目的。但由于智能刀具自身结 构及系统的复杂性, 其使用及维护需要专业人员进 行操作, 并且部分技术不成熟, 这都给智能刀具在 实际生产中的应用带来诸多问题, 如何推进智能刀 具在企业加工现场的实际应用, 需要高校研究人员 与企业管理者进行深入沟通与探索, 产学研互相协 作, 推进技术落地, 最终使研究人员的论文写在生 产一线上。

\section{参 考 文 献}

[1] 刘献礼, 刘强, 岳彩旭, 等. 切削过程中的智能技术 [J]. 机械工程学报, 2018, 54(16): 45-61.

LIU Xianli, LIU Qiang, YUE Caixu, et al. Intelligent technology in the cutting process[J]. Journal of Mechanical Engineering, 2018, 54(16): 45-61.

[2] 刘战强, 黄传真. 切削温度测量方法综述 [J]. 工具技 术, 2002, 36(3): 3-6.

LIU Zhanqiang, HUANG Chuanzhen. Summary of cutting temperature measurement methods[J]. Tool technology, 2002, 36(3): 3-6.

[3] 于启勋, 朱正芳. 刀具材料的历史、进展与展望[J]. 机 械工程学报, 2003, 39(12): 62-66.

YU Qixun, ZHU Zhengfang. The history、 progress and prospect of tool materials[J]. Journal of Mechanical Engineering, 2003, 39(12): 62-66.

[4] 蔡飞, 高营, 蔡习军, 等. 硬质合金刀具高能离子源增 强多弧镀 $\mathrm{AlCrTiSiN}$ 梯度涂层制备及性能研究[J]. 机 械工程学报, 2019，55(19): 213-220.

CAI Fei, GAO Ying, CAI Xijun, et al. Preparation and properties of multi-arc AlCrTiSiN gradient coating enhanced by high-energy ion source for cemented carbide tools[J]. Journal of Mechanical Engineering, 2019, 55(19) : 213-220.

[5] 刘日韦, 唐健, 贺连梁, 等. 刀具角度对 TC4 钢合金 切削力的影响[J]. 工具技术, 2014, 48(5): 17-20.

LIU Riwei, TANG Jian, HE Lianliang, et al. The influence of tool angle on cutting force of TC4 titanium alloy[J]. Tool Technology, 2014, 48(5): 17-20.

[6] 刘献礼, 范梦超, 计伟, 等. 椭球头铣刀设计及其刀具 路径生成算法 [J]. 机械工程学报, 2018, 54(15): 199-212.

LIU Xianli, FAN Mengchao, JI Wei, et al. Ellipsoid end milling cutter design and its tool path generation algorithm[J]. Journal of Mechanical Engineering, 2018, 54(15): 199-212.

[7] 李亚, 黄亦翔, 赵路杰, 等. 基于 $\mathrm{t}$ 分布邻域嵌入与 XGBoost 的刀具多工况磨损评估[J]. 机械工程学报, 2020, 56(01): 132-140.

LI Ya, HUANG Yixiang, ZHAO Lujie, et al. Multi-condition tool wear evaluation based on t-distribution neighborhood embedding and XGBoost[J]. Journal of Mechanical Engineering, 2020, 56(01): 132-140.

[8] 王国锋, 李志猛, 董毅. 刀具状态智能监测研究进展 [J]. 航空制造技术，2018，61(6): 16-23.

WANG Guofeng, LI Zhimeng, DONG Yi. Recent advances in intelligent monitoring of cutting tool condition[J]. Aviation Manufacturing Technology, 2018, 61(6): 16-23.

[9] 刘志军, 全燕鸣. 刀具切削状态监控技术综述 [J]. 工具 技术，2015，49(6): 3-7.

LIU Zhijun, QUAN Yanming. Overview of tool cutting condition monitoring technology[J]. Tool Technology, 2015, 49(6): 3-7.

[10] 成云平. 刀具嵌入式薄膜微传感器切削力测量技术的 基础研究[D]. 太原: 中北大学, 2015 .

CHENG Yunping. Basic research of embedded thin film micro sensor on cutting force measurement technology[D]. 
Taiyuan: North China University, 2015.

[11] 姚英学, 陈朔冬. 一种新结构多用途切削测力仪的研制 [J]. 哈尔滨工业大学学报, 1994, 26(4): 85-89.

YAO Yingxue, CHEN Shuodong. Development of a multipurpose machine tool dynamometer with new structure[J]. Journal of Harbin University of Technology, 1994, 26(4): $85-89$

[12] 孟萌. 嵌入薄膜微传感器测量切削力刀具的设计及试 验研究[D]. 太原: 中北大学, 2018.

MENG Meng. The design and experimental study of measuring cutting force tool with embedded thin film microsensor[D]. Taiyuan: North China University, 2018.

[13] 刘玉香. 切削力测量系统传感器设计[D]. 沈阳: 沈阳理 工大学, 2012.

LIU Yuxiang. The design of sensor on cutting force measurement system[D]. Shenyang: Shenyang Ligong University, 2012.

[14] 张铁. 三向压电式车削测力仪的性能研究与结构设计 [D]. 大连: 大连理工大学, 2007.

ZHANG Tie. Study and structure design on three-dimentional dynamometer of piezoelectricity on turning[D]. Dalian: Dalian University of Technology, 2007.

[15] KIM I H. Dynamic cutting force on-line estimation using a 4-electrode cylindrical capacitive displacement sensor mounted on a high speed milling spindle[J]. Journal of Mechanical Science \& Technology, 2008, 22(5): 914-923.

[16] 李文德. 基于声表面波原理的智能刀具系统关键技术 研究[D]. 哈尔滨: 哈尔滨工业大学, 2013.

LI Wende. Research on the key technologies about smart cutting tool system based on surface acoustic wave techniques[D]. Harbin: Harbin Institute of Technology, 2013.

[17] YALDZ S, ÜNSAAR F. A dynamometer design for measurement the cutting forces on turning $[\mathrm{J}]$. Measurement, 2006, 39(1): 80-89.

[18] PANZERA T H, SOUZA P R. Development of a three-component dynamometer to measure turning force[J]. International Journal of Advanced Manufacturing Technology, 2012, 62(9-12): 913-922.

[19] ZHAO You, ZHAO Yulong, LIANG Songbo, et al. A high performance sensor for triaxial cutting force measurement in turning[J]. Sensors, 2015, 15(4) : 7969-7984.

[20] 赵友, 葛晓慧, 赵玉龙. 高精度动态切削力自感知智能
刀具的研究 [J]. 机械工程学报, 2019, 55(21): 178-185. ZHAO You, GE Xiaohui, ZHAO Yulong. Research on high-precision dynamic cutting force self-sensing intelligent tool[J]. Journal of Mechanical Engineering, 2019, 55(21): 178-185.

[21] HARMON A, FUSSELL B K, JERARD R B. Calibration and characterization of a low-cost wireless sensor for applications in $\mathrm{CNC}$ end milling[C]//ASME International Manufacturing Science \& Engineering Conference Collocated with the North American Manufacturing Research Conference \& in Participation with the International Conference on Tribology Materials \& Processing, 2012: 823-832.

[22] RIZAL M , GHANI J A , NUAWI M Z, et al. Development and testing of an integrated rotating dynamometer on tool holder for milling process[J]. Mechanical Systems and Signal Processing, 2015, 52-53: 559-576.

[23] SUPROCK C A, NICHOLS J S. A low cost wireless high bandwidth transmitter for sensor-integrated metal cutting tools and process monitoring[J]. International Journal of Mechatronics and Manufacturing Systems, 2009, 2(4): 441-454.

[24] 徐佳成. 超声加工压电式三向测力仪的关键技术研究[D]. 杭州: 杭州电子科技大学, 2018 .

XU Jiacheng. Research on key techniques of ultrasonic piezoelectric three-dimensional force gauge[D]. Hangzhou: Hangzhou University of Electronic Science and Technology, 2018 .

[25] 孙宝元, 张贻恭, 杨华敏. 压电式动态切削测力仪的研 究(上) $[\mathrm{J}]$. 机床, 1979(5): 1-5.

SUN Baoyuan, ZHANG Yigong, YANG Huamin. Piezoelectric dynamic cutting dynamometer (part 1)[J]. Machine Tool, 1979(5): 1-5.

[26] 孙宝元, 张贻恭, 张松涛. 压电式动态切削测力仪的 研究(下)[J]. 机床, 1979(10): 27-33.

SUN Baoyuan, ZHANG Yigong, ZHANG Songtao. Piezoelectric dynamic cutting dynamometer (part 2)[J]. Machine Tool, 1979(10): 27-33.

[27] 孙宝元, 张贻恭. 压电石英力传感器及动态切削测力仪 $[\mathrm{M}]$. 北京: 计量出版社, 1985.

SUN Baoyuan, ZHANG Yigong. Piezoelectric quartz force sensor and dynamic cutting dynamometer[M]. Beijing: Metrology Press, 1985.

[28] 孙宝元. 三向压电式车削测力仪结构的优化设计 [J]. 
机械工程学报, 1990, 26(2): 65-72.

SUN Baoyuan. Optimal design of the structure of the three-way piezoelectric turning dynamometer[J]. Journal of Mechanical Engineering， 1990，26(2): 65-72.

[29] 肖才伟. 基于切削力感知的智能切削刀具设计及其关 键技术研究[D]. 哈尔滨: 哈尔滨工业大学, 2014.

XIAO Caiwei. Design and key technologies research of smart cutting tool based on cutting force sensing[D]. Harbin: Harbin Institute of Technology, 2014.

[30] TOTIS G, SORTINO M. Development of a modular dynamometer for triaxial cutting force measurement in turning $[\mathrm{J}]$. International Journal of Machine Tools and Manufacture, 2011, 51(1): 34-42.

[31] TOTIS G, WIRTZ G, SORTINO M, et al. Development of a dynamometer for measuring individual cutting-edge forces in face milling[J]. Mechanical Systems and Signal Processing, 2010, 24(6): 1844-1857.

[32] REN Zongjin, ZHANG Jun, JIA Zhenyuan, et al. Design and calibration of a cutting force dynamometer[J]. Advanced Materials Research, 2014, 945-949: 2191-2194.

[33] 尚永艳. 刀柄式压电切削测力仪研究 $[D]$. 大连: 大连理 工大学, 2014.

SHANG Yongyan. Research on tool holder piezoelectric cutting dynamometer[D]. Dalian: Dalian University of Technology, 2014.

[34] ZHENG L, RAMALINGAM S, SHI T, et al. Aluminum nitride thin film sensor for force, acceleration, and acoustic emission sensing[J]. Journal of Vacuum Science \& Technology A Vacuum Surfaces and Films, 1993, 11(5): 2437-2446.

[35] WANG Chao, RAWKOSKI R, CHENG Kai. Design and analysis of a piezoelectric film embedded smart cutting tool[J]. Proceedings of the Institution of Mechanical Engineers, Part B: Journal of Engineering Manufacture, 2013, 227(2): 254-260.

[36] CHENG Kai, NIU Zhichao, WANG R C, et al. Smart Cutting Tools and Smart Machining: Development Approaches, and Their Implementation and Application Perspectives[J]. Chinese Journal of Mechanical Engineering, 2017(5): 1162-1176.

[37] LUO Ming, LUO Huan, AXINTE D, et al. A wireless instrumented milling cutter system with embedded PVDF sensors[J]. Mechanical Systems and Signal Processing, 2018, 110: 556-568.

[38] MA L, MELKOTE S N, Castle J B. PVDF sensor-based monitoring of milling torque[J]. The International Journal of Advanced Manufacturing Technology, 2014, 70(9-12): 1603-1614.

[39] MA L, MELKOTE S N, MOREHOUSE J B, et al. thin-film PVDF sensor-based monitoring of cutting forces in peripheral end milling[J]. Journal of Dynamic Systems, Measurement, and Control, 2012, 134(5): 1-9.

[40] 郝悦. 电容式四维测力刀柄性能测试技术研究 [D]. 哈 尔滨：哈尔滨工业大学, 2016.

HAO Yue. Research on performance testing for capacitive four-dimensional force-measuring tool holder[D]. Harbin: Harbin Institute of Technology, 2016.

[41] ALBRECHT A, PARK S S, ALTINTAS Y, et al. High frequency bandwidth cutting force measurement in milling using capacitance displacement sensors[J]. International Journal of Machine Tools and Manufacture, 2005, 45(9): 993-1008.

[42] KIM J H, CHANG H K, HAN D C, et al. Cutting force estimation by measuring spindle displacement in milling process[J]. CIRP Annals Manufacturing Technology, 2005, 54(1): 67-70.

[43] STONEY R, O'DONNELL G, EGERAGHTY D. Dynamic wireless passive strain measurement in CNC turning using surface acoustic wave sensors[J]. International Journal of Advanced Manufacturing Technology, 2013, 69(5-8): 1421-1430.

[44] STONEY R, DONOHOE B, GERAGHTY D, et al. The development of surface acoustic wave sensors (SAWs) for Process Monitoring[J]. Procedia CIRP, 2012, 1: 569-574.

[45] 李文德, 丁辉, 程凯. 基于声表面波原理的切削力测量 智能刀具研究 $[J]$. 机械制造与自动化, 2014(5): 46-50. LI Wende, DING Hui, CHENG Kai. Research on force measuring smart cutting tool based on surface acoustic wave resonator principle $[\mathrm{J}]$. Machinery Manufacturing and Automation, 2014(5): 46-50.

[46] WANG Chao, CHENG Kai, CHEN Xun, et al. Design of an instrumented smart cutting tool and its implementation and application perspectives[J]. Smart Materials and Structures, 2014, 23(3): 623-626.

[47] 王震宇. 高速立铣切削刀具温度场建模与实时在线温 度测量技术研究 $[D]$. 哈尔滨: 哈尔滨理工大学, 2015 . WANG Zhenyu. Study on the temperature field modeling and the real-time online temperature measuring technique for the high speed end mill[D]. Harbin: Harbin University of Technology, 2015. 
[48] 徐念伟, 付秀丽, 郝宗成, 等. 金属切削加工瞬态温度 测量研究综述 $[J]$. 工具技术，2019，53(2)：5-9.

XU Nianwei, FU Xiuli, HAO Zongcheng, et al. Summary of transient temperature measurement in metal cutting[J]. Tool Technology, 2019, 53(2): 5-9.

[49] SUGITA N, ISHII K, FURUSHO T, et al. Cutting temperature measurement by a micro-sensor array integrated on the rake face of a cutting tool[J]. CIRP Annals - Manufacturing Technology, 2015, 64(1): 77-80.

[50] 崔云先, 张博文, 丁万昱, 等. 瞬态切削用智能测温刀 具的研究 [J]. 机械工程学报, 2017(21): 185-191. CUI Yunxian, ZHANG Bowen, DING Wanyu, et al. Research on the cutting tool with intelligent transient temperature measuring system[J]. Journal of Mechanical Engineering, 2017(21): 185-191.

[51] 李林文. 面向硬切削的切削区域温度场解析建模及实 验研究[D]. 武汉: 华中科技大学, 2013.

LI Linwen. Analytical modeling and experimental study of temperature field in cutting area for hard cutting [D]. Wuhan : Huazhong University of Science and Technology, 2013.

[52] SHUWEN H, BO T, JINDANG L, et al. Estimation of the time and space-dependent heat flux distribution at the tool-chip interface during turning using an inverse method and thin film thermocouples measurement[J]. International Journal of Advanced Manufacturing Technology, 2018, 99: 1531-1543.

[53] SHU Shengrong, DING Hui, CHEN Shijin, et al. FEM-based design and analysis of a smart cutting tool with internal cooling for cutting temperature measurement and control[J]. Applied Mechanics and Materials, 2012, 217-219: 1874-1879.

[54] COZ G L, MARINESCU M, DEVILLEZ A, et al. Measuring temperature of rotating cutting tools : Application to MQL drilling and dry milling of aerospace alloys[J]. Applied Thermal Engineering, 2012, 36: 434-441.

[55] WRIGHT P K, DORNFELD D A, HILLAIRE R G, et al. A wireless sensor for tool temperature measurement and its integration within a manufacturing system[J]. Transactions of the North American Manufacturing Research Institute of SME，2006，34: 63-70.

[56] KERRIGAN K, O'DONNELL G E. Temperature measurement in CFRP milling using a wireless tool-integrated process monitoring sensor[J]. International
Journal of Automation Technology, 2013, 7(6): 742-750.

[57] DEVILLEZ A, DUDZINSKI D. Tool vibration detection with eddy current sensors in machining process and computation of stability Lobes using fuzzy classifiers[J]. Mechanical Systems \& Signal Processing, 2007, 21(1): 441-456.

[58] CHUNG T K, YEH P C, HAO L, et al. An attachable electromagnetic energy harvester driven wireless sensing system demonstrating milling-processes and cutter-wear/breakage-condition monitoring[J]. Sensors, 2016, 16(3): 1-18.

[59] CHUNG T, LEE H, TSENG C, et al. Self-powered wireless vibration-sensing system for machining monitoring[C]// Proceedings of SPIE-The International Society for Optical Engineering. San Diego, CA, United states: SPIE, 2013, 8692 86922U-1.

[60] PENG Huanghu, WU Yijie, WANG Bin, et al. An improved two-point real-time measuring method for radial micro-displacement measurement on high-speed smart boring bar[J]. The International Journal of Advanced Manufacturing Technology, 2015, 81(5-8): 925-933.

[61] SUPROCK C A, FUSSELL B K, HASSAN R Z, et al. A low cost wireless tool tip vibration sensor for milling[C]//ASME International Manufacturing Science \& Engineering Conference Collocated with the JSME/ASME International Conference on Materials \& Processing. 2008.

[62] 刘海军. 面向铣削过程的无线测振刀柄的关键技术研 究[D]. 哈尔滨：哈尔滨工业大学, 2015.

LIU Haijun. Research for key technology of vibration-detecting and wireless-transmitting tool holder in milling process[D]. Harbin : Harbin Institute of Technology, 2015.

[63] GRANADOS G H, MORITA N, HIDAI H, et al. Development of a non-rigid micro-scale cutting mechanism applying a normal cutting force control system[J]. Precision Engineering, 2016， 43: 544-553.

[64] LI H, IBRAHIM R, CHENG K. Design and principles of an innovative compliant fast tool servo for precision engineering[J]. Mech. Sci, 2011, 2:139-146.

[65] CHENG Kai, NIU Zhichao, WANG R C, et al. Smart cutting tools and smart machining: Development approaches, and their implementation and application perspectives $[\mathrm{J}]$. Chinese Journal of Mechanical 
Engineering, 2017, 30: 1162-1176.

[66] KURIYAMA K, FUKUTA M, SEKIYA K, et al. Applying constant pressure unit to ductile mode cutting of hard and brittle materials[J]. International Journal of Automation Technology, 2013, 7(3): 278-284.

[67] 舒盛荣. 内冷式智能车刀设计与分析及其实验研究 $[D]$. 哈尔滨：哈尔滨工业大学, 2014.

SHU Shengrong. The design and analysis of the internally cooled intelligent turning tool and its experimental research [D]. Harbin: Harbin Institute of Technology, 2014.

[68] 刘文博, 李天箭, 石永泉, 等. 基于 LabVIEW 的内冷 式智能车刀温度控制系统的设计 $[\mathrm{J}]$. 农业装备与车辆 工程, 2018, 56(10): 10-12.

LIU Wenbo, LI Tianjian, SHI Yongquan, et al. The design of the temperature control system of the inner-cooled intelligent turning tool based on LabVIEW[J]. Agricultural Equipment and Vehicle Engineering, 2018, 56(10): 10-12.

[69] SUN X, BATEMAN R, CHENG K, et al. Design and analysis of an internally cooled smart cutting tool for dry cutting $[\mathrm{J}]$. Proceedings of the Institution of Mechanical Engineers Part B Journal of Engineering Manufacture, 2011, 226(4): 585-591.

[70] 梁良. 面向绿色切削的热管刀具散热性能研究[D]. 广 州: 华南理工大学, 2011 .

LIANG Liang. Research on heat dissipation performance of heat pipe cutters for green cutting[D]. Guangzhou: South China University of Technology, 2011.

[71] LIANG Liang, QUAN Yanming. Investigation of heat partition in dry turning assisted by heat pipe cooling $[\mathrm{J}]$. International Journal of Advanced Manufacturing Technology, 2013, 66(9-12): 1931-1941.

[72] LU Xiaodong, CHEN Fan, ALTINTAS Y. Magnetic actuator for active damping of boring bars[J]. CIRP Annals-Manufacturing Technology, 2014, 63(1): 369-372.

[73] CHEN Fan, LU Xiaodong, ALTINTAS Y. A novel magnetic actuator design for active damping of machining tools[J]. International Journal of Machine Tools and Manufacture, 2014, 85: 58-69.

[74] CHEN Fan, LIU Guangya. Active damping of machine tool vibrations and cutting force measurement with a magnetic actuator[J]. International Journal of Advanced Manufacturing Technology, 2017, 89(1-4): 691-700.

[75] CHEN Fan, HANIFZADEGAN M , ALTINTAS Y, et al. Active damping of boring bar vibration with a magnetic actuator[J]. IEEE/ASME Transactions on Mechatronics, 2015: 1-12.

[76] LIU Xianli, LIU Qiang, WU Shi, et al. Research on the performance of damping boring bar with a variable stiffness dynamic vibration absorber[J]. International Journal of Advanced Manufacturing Technology, 2017, 89(9-12): 2893-2906.

[77] MATSUBARA A, MAEDA M, YAMAJI I. Vibration suppression of boring bar by piezoelectric actuators and LR circuit[J]. CIRP Annals - Manufacturing Technology, 2014, 63(1): 373-376.

[78] 王民, 费仁元. 切削系统可变刚度结构及其颤振控制方 法的研究 [J]. 机械工程学报, 2002, 38(增): 219-222.

WANG Min, FEI Renyuan. Research of variable-stiffness structure and varying stiffness method on chatter control[J]. Journal of Mechanical Engineering, 2002, 38(Suppl.): 219-222.

[79] 王民, 费仁元. 基于电流变材料的切削颤振在线监控技 术研究 [J]. 机械工程学报, 2002，38(12): 93-97.

WANG Min, FEI Renyuan. Research on monitored control of machining chatterbased on electrorheological fluids[J]. Journal of Mechanical Engineering, 2002, 38(12): 93-97.

[80] MEI Deqing, YAO Zhehe, KONG Tianrong, et al. Parameter optimization of time-varying stiffness method for chatter suppression based on magnetorheological fluid-controlled boring bar[J]. International Journal of Advanced Manufacturing Technology, 2010， 46(9-12): 1071-1083.

[81] YAO Zhehe, MEI Deqing, CHEN Zichen. Chatter suppression by parametric excitation: Model and experiments[J]. Journal of Sound \& Vibration, 2011, 330(13): 2995-3005.

[82] 孔天荣. 磁流变自抑振智能镗杆的理论与方法研究 [D]. 杭州: 浙江大学, 2009.

KONG Tianrong. Research on theory and method of magnetorheological self damping intelligent boring bar [D]. Hangzhou: Zhejiang University, 2009.

[83] 孔天荣, 梅德庆, 陈子辰. 磁流变智能镗杆的动态特性 测试与分析 [J]. 浙江大学学报, 2009, 43(12): 2314-2318.

KONG Tianrong, MEI Deqing, CHEN Zichen. Dynamic characteristics test and analysis of magnetorheological intelligent boring bar [J]. Journal of Zhejiang University, 
2009, 43 (12): 2314-2318.

[84] 孔天荣, 梅德庆, 陈子辰. 磁流变智能镗杆的切削颤振 抑制机理研究 [J]. 浙江大学学报, 2008, 42(6): 1005-1009.

KONG Tianrong, MEI Deqing, CHEN Zichen. Research on mechanism of cutting chatter suppression based onmagnetorheological intelligent boring bar[J]. Journal of Zhejiang University, 2008, 42(6): 1005-1009.

[85] 李欣. 基于 HMM-SVM 的磁流变自抑振智能镗杆 颤振在线预报理论和方法研究 [D]. 杭州: 浙江大 学, 2013.

LI Xin. Research on theory and method of online prediction of intelligent boring bar chatter based on HMM-SVM [D]. Hangzhou: Zhejiang University, 2013.
[86] 李欣, 梅德庆, 陈子辰. 基于经验模态分解和希尔伯特黄变换的精密孔镗削颤振特征提取 [J]. 光学精密工程, 2011, 19(6): 1291-1297.

LI Xin, MEI Deqing, CHEN Zichen. Chatter feature extraction of precision hole boring based on empirical mode decomposition and Hilbert-Huang transform [J]. Optical Precision Engineering, 2011, 19(6): 1291-1297.

[87] MONNIN J, KUSTER F, WEGENER K, et al. Optimal control for chatter mitigation in milling-Part 1: Modeling and control design[J]. Control Engineering Practice, 2014, 24: 156-166.

作者简介: 刘强(通信作者), 男, 1988 年出生, 博士, 硕士研究生导师。 主要研究方向为智能刀具技术基础及应用。

E-mail: liuqianglink@163.com 